University of Wollongong

Research Online

Australian Institute for Innovative Materials -

Papers

Australian Institute for Innovative Materials

$1-1-2020$

Diffused morphotropic phase boundary in relaxor-PbTiO3crystals: High piezoelectricity with improved thermal stability

Gang Liu

Lingping Kong

Qingyang Hu

Shujun Zhang

University of Wollongong, shujun@uow.edu.au

Follow this and additional works at: https://ro.uow.edu.au/aiimpapers

Part of the Engineering Commons, and the Physical Sciences and Mathematics Commons

Research Online is the open access institutional repository for the University of Wollongong. For further information contact the UOW Library: research-pubs@uow.edu.au 


\title{
Diffused morphotropic phase boundary in relaxor-PbTiO3crystals: High piezoelectricity with improved thermal stability
}

\author{
Abstract \\ (C) 2020 Author(s). Solid solution ferroelectrics are the most widely used piezoelectric material for \\ numerous electromechanical applications, including sensors, actuators, and transducers. A milestone in \\ ferroelectric research is the discovery of the morphotropic phase boundary that was first reported in \\ $\mathrm{Pb}(\mathrm{ZrxTi1-x}) 03$, which has been extensively solicited to improve the performance of various solid solution \\ ferroelectrics, including those having the highest piezoelectricity known today. However, due to the \\ inherent correlation between phase transition and thermodynamic imbalance, the efforts of building the \\ phase boundary encounter the challenge that high performance materials are generally accompanied by \\ property instability. Here, we report a comprehensive study on the crystalline symmetry and polar nano- \\ regions in relaxor-PbTiO3 crystals by synchrotron measurements and property characterizations. In \\ contrast to the common belief that the morphotropic phase boundary is a narrow composition region, the \\ morphotropic phase boundary in ternary $\mathrm{Pb}(\operatorname{In} 1 / 2 \mathrm{Nb} 1 / 2) 03-\mathrm{Pb}(\mathrm{Mg} 1 / 3 \mathrm{Nb} 2 / 3) 03-\mathrm{PbTiO} 3$ is demonstrated \\ as an extensive region covering at least $9 \%$ of the $\mathrm{PbTiO} 3$ content. Such a diffused morphotropic phase \\ boundary is associated with the intensive interaction of polar nano-regions, leading to high \\ piezoelectricity $(>1500 \mathrm{pC} / \mathrm{N})$ with greatly improved thermal stability, where the piezoelectric variation is \\ $\sim 90 \%$ over the temperature range of $273-373 \mathrm{~K}$, which is about a factor of 3 lower compared to its binary \\ counterpart $\mathrm{Pb}(\mathrm{Mg} 1 / 3 \mathrm{Nb} 2 / 3) 03-\mathrm{PbTiO}$. This work sheds light on the fundamental understanding of \\ nanoscale inhomogeneity and macroscopic symmetry in relaxor-PbTiO3, and successfully interlinks the \\ structure and properties in complex solid solutions. The existence of a diffused morphotropic phase \\ boundary is also expected to benefit other ferroic systems beyond ferroelectrics, such as ferromagnetic \\ and multiferroic materials. \\ Disciplines \\ Engineering | Physical Sciences and Mathematics

\section{Publication Details} \\ Liu, G., Kong, L., Hu, Q. \& Zhang, S. (2020). Diffused morphotropic phase boundary in relaxor- \\ PbTiO3crystals: High piezoelectricity with improved thermal stability. Applied Physics Reviews, 7 (2), \\ 021405-1-021405-12.
}




\section{Diffused morphotropic phase boundary in relaxor- $\mathrm{PbTiO}_{3}$ crystals: High piezoelectricity with improved thermal stability}

Cite as: Appl. Phys. Rev. 7, 021405 (2020); https://doi.org/10.1063/5.0004324

Submitted: 10 February 2020 . Accepted: 14 April 2020 . Published Online: 07 May 2020

Gang Liu, Lingping Kong, Qingyang Hu, and Shujun Zhang

\section{COLLECTIONS}

F This paper was selected as Featured
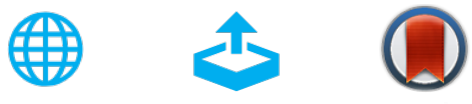

\section{ARTICLES YOU MAY BE INTERESTED IN}

Genesis and evolution of extended defects: The role of evolving interface instabilities in cubic $\mathrm{SiC}$

Applied Physics Reviews 7, 021402 (2020); https://doi.org/10.1063/1.5132300

Identification of light sources using machine learning

Applied Physics Reviews 7, 021404 (2020); https://doi.org/10.1063/1.5133846

Broadband enhancement of on-chip single-photon extraction via tilted hyperbolic metamaterials

Applied Physics Reviews 7, 021403 (2020); https://doi.org/10.1063/1.5141275

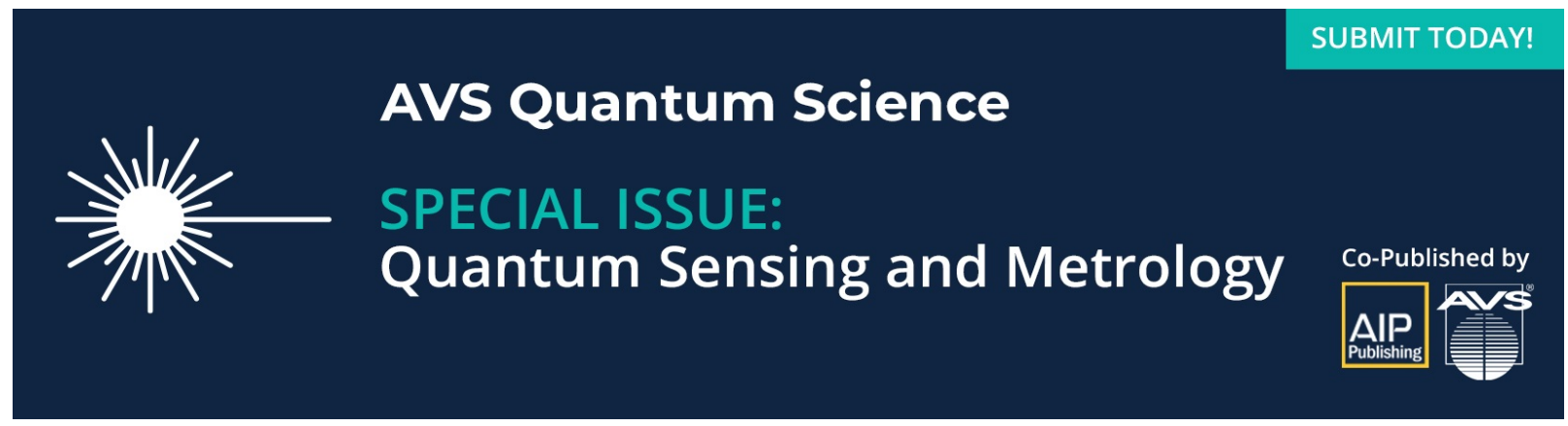




\title{
Diffused morphotropic phase boundary in relaxor- $\mathrm{PbTiO}_{3}$ crystals: High piezoelectricity with improved thermal stability

Cite as: Appl. Phys. Rev. 7, 021405 (2020); doi: 10.1063/5.0004324

Submitted: 10 February 2020 - Accepted: 14 April 2020 •

Published Online: 7 May 2020

Gang Liu, ${ }^{1, a)}$ Lingping Kong, ${ }^{1, a)}$ Qingyang Hu, ${ }^{7}$ and Shujun Zhang ${ }^{2, a)}$

\begin{abstract}
AFFILIATIONS
${ }^{7}$ Center for High Pressure Science and Technology Advanced Research, Shanghai 201203, China

${ }^{2}$ Institute for Superconducting and Electronic Materials, Australian Institute for Innovative Materials, University of Wollongong, NSW 2500, Australia

${ }^{a)}$ Authors to whom correspondence should be addressed: liugang@hpstar.ac.cn; konglp@hpstar.ac.cn; and shujun@uow.edu.au
\end{abstract}

\begin{abstract}
Solid solution ferroelectrics are the most widely used piezoelectric material for numerous electromechanical applications, including sensors, actuators, and transducers. A milestone in ferroelectric research is the discovery of the morphotropic phase boundary that was first reported in $\mathrm{Pb}\left(\mathrm{Zr}_{\mathrm{x}} \mathrm{Ti}_{1-\mathrm{x}}\right) \mathrm{O}_{3}$, which has been extensively solicited to improve the performance of various solid solution ferroelectrics, including those having the highest piezoelectricity known today. However, due to the inherent correlation between phase transition and thermodynamic imbalance, the efforts of building the phase boundary encounter the challenge that high performance materials are generally accompanied by property instability. Here, we report a comprehensive study on the crystalline symmetry and polar nano-regions in relaxor- $\mathrm{PbTiO}_{3}$ crystals by synchrotron measurements and property characterizations. In contrast to the common belief that the morphotropic phase boundary is a narrow composition region, the morphotropic phase boundary in ternary $\mathrm{Pb}\left(\mathrm{In}_{1 / 2} \mathrm{Nb}_{1 / 2}\right) \mathrm{O}_{3}-\mathrm{Pb}\left(\mathrm{Mg}_{1 / 3} \mathrm{Nb}_{2 / 3}\right) \mathrm{O}_{3}-\mathrm{PbTiO}_{3}$ is demonstrated as an extensive region covering at least $9 \%$ of the $\mathrm{PbTiO}_{3}$ content. Such a diffused morphotropic phase boundary is associated with the intensive interaction of polar nano-regions, leading to high piezoelectricity $(>1500 \mathrm{pC} / \mathrm{N})$ with greatly improved thermal stability, where the piezoelectric variation is $\sim 90 \%$ over the temperature range of $273-373 \mathrm{~K}$, which is about a factor of 3 lower compared to its binary counterpart $\mathrm{Pb}\left(\mathrm{Mg}_{1 / 3} \mathrm{Nb}_{2 / 3}\right) \mathrm{O}_{3}-\mathrm{PbTiO}_{3}$. This work sheds light on the fundamental understanding of nanoscale inhomogeneity and macroscopic symmetry in relaxor- $\mathrm{PbTiO}_{3}$, and successfully interlinks the structure and properties in complex solid solutions. The existence of a diffused morphotropic phase boundary is also expected to benefit other ferroic systems beyond ferroelectrics, such as ferromagnetic and multiferroic materials.
\end{abstract}

Published under license by AIP Publishing. https://doi.org/10.1063/5.0004324

\section{INTRODUCTION}

The concept of morphotropic phase boundary (MPB) was developed to demonstrate the composition dependent ferroelectricferroelectric phase transitions, which is the mainstay approach to improve the performance of ferroelectric solid solutions and guides the design of new piezoelectric materials. ${ }^{1-4}$ The MPB was initially reported to be a linear boundary in $\mathrm{Pb}\left(\mathrm{Zr}_{x} \mathrm{Ti}_{1-x}\right) \mathrm{O}_{3}(\mathrm{PZT}){ }^{5}$, separating rhombohedral and tetragonal phases, which possess similar free energies. In the late 1990s, the monoclinic phases were observed in $\mathrm{PbZr}_{0.52} \mathrm{Ti}_{0.48} \mathrm{O}_{3}$ by synchrotron XRD. ${ }^{6}$ These low-symmetry monoclinic phases were thought to behave as the bridging phases connecting rhombohedral and tetragonal structures, ${ }^{7,8}$ thus expanding the MPB region in the composition-temperature phase diagram, further flattening the free energy profile of the polarization rotation between rhombohedral and tetragonal phases, ${ }^{9}$ and being responsible for the enhanced piezoelectricity. Therefore, it is generally accepted that the MPB is a phase coexistence region, where the composition width of MPB is crucial for functionality improvement. The MPB concept is particularly important also because it has been expanded to ferromagnetics, multiferroics, and functional alloys, all of which describe the unique properties due to the energy competition between two parent crystal phases, ${ }^{10-12}$ further confirming the strong correlation between high functionalities and structural instability.

Analogous to ferroelectric-ferroelectric MPB, obvious piezoelectric enhancement has also been demonstrated as the temperature approaches the ferroelectric-paraelectric phase transition, where the 
crystallographic structure becomes more cubic-like and the freeenergy profile is globally isotropic. Thus, an easier polarization extension occurs along the direction linking the paraelectric phase (cubic) and ferroelectrics phase. As an example, a high piezoelectric coefficient, $\sim 620 \mathrm{pC} / \mathrm{N}$, has been reported in $\mathrm{Ba}\left(\mathrm{Ti}_{0.8} \mathrm{Zr}_{0.2}\right) \mathrm{O}_{3}-\left(\mathrm{Ba}_{0.7} \mathrm{Ca}_{0.3}\right)$ $\mathrm{TiO}_{3}$ (BZT-BCT) lead-free piezoceramics with a relatively low Curie temperature of $\sim 100^{\circ} \mathrm{C}$, which is associated with the critical triple phases. ${ }^{13}$ It should be noted that high piezoelectric coefficients in temperature-driven phase transition regions are rarely useful because of the inherent instabilities. ${ }^{14}$

To further improve the piezoelectric activity of ferroelectric materials, two general strategies have been employed, including the design of ferroelectric solid solutions with MPB composition and a shift in the polymorphic phase transition near room temperature [Fig. 1(a)]. Due to the inevitable phase transitions and/or decreased Curie temperature, these efforts encounter the challenge that high-performance materials are generally accompanied by instabilities [Figs. 1(b) and 1 (c)], including the considerable loss of thermal resistance ability, and thus exhibit significant variation in properties as a function of temperature. Therefore, it is desired to re-scrutinize the properties of the solid solution ferroelectrics to gain a fundamental understanding of phase transition boundary, which is expected to broaden the usage temperature range while retaining, if not improving, the piezoelectric merits of solid solution ferroelectrics with MPB composition.

The most recently proposed approach to increase piezoelectricity in ferroelectrics is to engineer the local structure heterogeneity, which induces the greatly flattened local free-energy profile by interfacial energy competition. ${ }^{18}$ The local structure heterogeneity, or polar nanoregions, was first reported to exist in complex perovskites with the chemical formula of $\mathrm{Pb}\left(\mathrm{B}^{\prime}, \mathrm{B}^{\prime \prime}\right) \mathrm{O}_{3}$, named as relaxor ferroelectrics, ${ }^{19}$ where $\mathrm{B}^{\prime}$ and $\mathrm{B}^{\prime \prime}$ are low and high valence cations, respectively. These relaxor ferroelectrics have frequency-dispersed dielectric properties with diffused phase transition characteristics. Then, it was discovered that by creating a solid solution with normal ferroelectrics and a relaxor end member, the piezoelectric activity can be greatly improved with peak values at the MPB over a broad composition range, demonstrating a great potential for commercial applications. This phenomenon was empirically employed in $\mathrm{Pb}\left(\mathrm{Ni}_{1 / 3} \mathrm{Nb}_{2 / 3}\right) \mathrm{O}_{3^{-}}$ $\mathrm{Pb}\left(\mathrm{Zr}_{0.3} \mathrm{Ti}_{0.7}\right) \mathrm{O}_{3}$ (PNN-PZT) solid solution, ${ }^{20-23}$ where the coexistence of the rhombohedral and tetragonal phases over a composition range of 0.3PNN-0.7PZT and 0.1PNN-0.9PZT accounts for the high dielectric constant, ${ }^{23}$ thus suggesting the crucial role of a wide MPB in property improvement of ferroelectric ceramics. It is worth noting that the studied PNN-PZT samples are polycrystalline ceramics, in which the compositional homogeneity, sample processing conditions, and particle size will significantly affect the width of MPB. ${ }^{24-27}$ In addition, we should note that the detectable width of a MPB depends on the compositional/phase resolution of the characterization tools, and in this context, the employed in-house XRD cannot provide high enough resolution for accurate structural analysis. Therefore, it is essential to study single crystal systems with a high resolution XRD technique in order to comprehensively understand the role of the wide MPB region in solid solution ferroelectrics and its impact on macroscopic properties.

In the early 1970s, solid solution ferroelectric single crystal $\mathrm{Pb}\left(\mathrm{Zn}_{1 / 3} \mathrm{Nb}_{1 / 2}\right) \mathrm{O}_{3}-\mathrm{PbTiO}_{3}$ was reported, ${ }^{28}$ followed by the successful growth of various relaxor- $\mathrm{PbTiO}_{3}$ single crystals with ultrahigh piezoelectric coefficients and electromechanical coupling factors in the 1980s through the 1990s. ${ }^{29-32}$ The cornerstone ferroelectric research on relaxor- $\mathrm{PbTiO}_{3}$ perovskite solid solution with MPB compositions has been the driving force for emerging electromechanical applications, with the latest piezoelectric activity reaching over $4000 \mathrm{pC} / \mathrm{N}$ in Sm-doped $\mathrm{Pb}\left(\mathrm{Mg}_{1 / 3} \mathrm{Nb}_{2 / 3}\right) \mathrm{O}_{3}-\mathrm{PbTiO}_{3}$ (PMN-PT) single crystals. ${ }^{33}$ The actively studied relaxor-PT crystals possess a narrow MPB region with 2-3 mol. $\% \mathrm{PbTiO}_{3}$ consisting of different ferroelectric phases on both sides. ${ }^{34,35}$

In this work, we conducted a detailed structural analysis on the $\mathrm{Pb}\left(\mathrm{In}_{1 / 2} \mathrm{Nb}_{1 / 2}\right) \mathrm{O}_{3}-\mathrm{Pb}\left(\mathrm{Mg}_{1 / 3} \mathrm{Nb}_{2 / 3}\right) \mathrm{O}_{3}-\mathrm{PbTiO}_{3}$ (PIN-PMN-PT) ternary crystals and compared it to PMN-PT binary crystals using ultrahighresolution synchrotron-based characterizations. We discovered that the monoclinic crystallographic symmetry highly diffuses into both the rhombohedral and tetragonal phases with an extremely extended

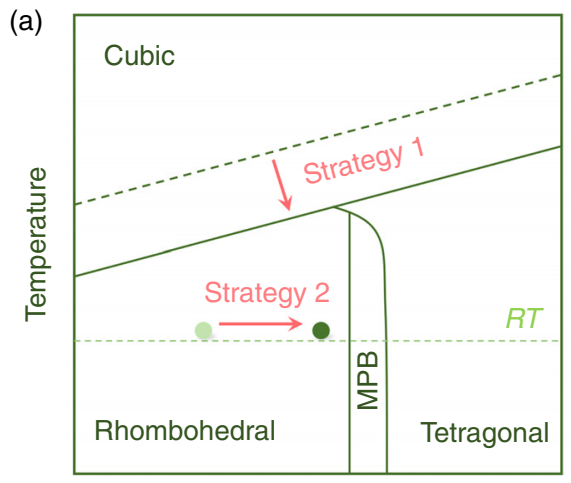

Relaxor

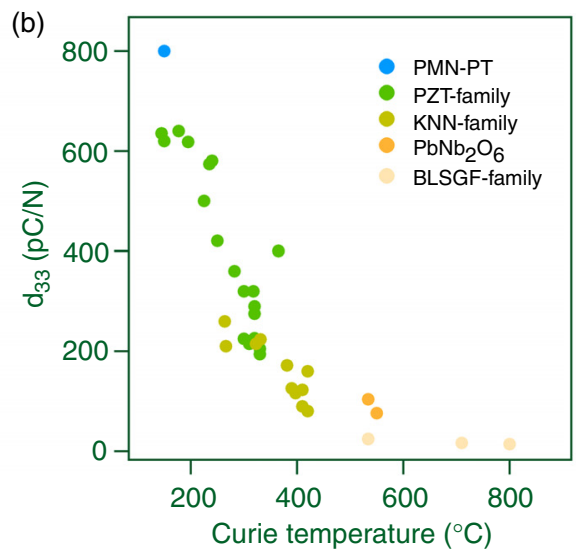

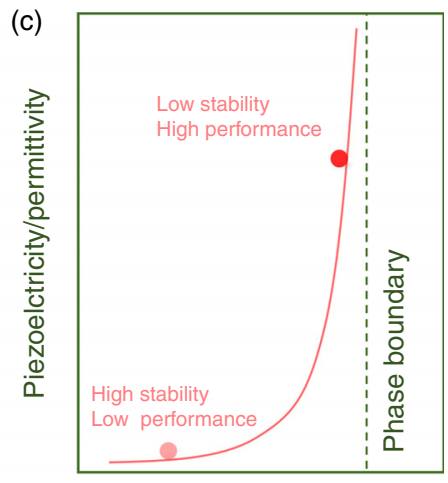

Temperature/composition

FIG. 1. The dilemma in ferroelectrics: high-performance is usually accompanied by thermodynamic instabilities. (a) Two strategies for high piezoelectric activity: lowering the Curie temperature (strategy 1) and approaching the MPB (strategy 2). (b) The relationship between the Curie temperature and the piezoelectric coefficient $d_{33}$ for various ferroelectric ceramics. Data are from Refs. 2 and 15-17. The high piezoelectric activity is clearly associated with lower Curie temperature. (c) High performance of ferroelectrics occurs at the phase boundary but creates thermodynamic instability. 
span of at least 9 mol. \% PT contents in the ternary solid solution. Our high-resolution synchrotron-based experiments, on the one hand, provided a solid proof for the diffused MPB in PIN-PMN-PT crystals, which quantitatively determined the width of the MPB region. Such a diffused MPB motivated us to replot the composition-temperature phase diagram and answered the question why both ultrahigh piezoelectricity and good thermal stability can be achieved simultaneously in the relaxor ferroelectric system. On the other hand, the obtained structural information, along with the detailed characterizations of dielectric/piezoelectric behavior, clarified the underlying mechanism linking the local structure at the nanoscale, the crystallographic symmetry, and the macroscopic properties in relaxor-PT crystals, which also echoes previous observations in PZT-based ceramics. ${ }^{23}$

\section{RESULTS AND DISCUSSION}

\section{A. Crystal samples and functional characterizations}

High-quality [001] grown PIN-PMN-PT crystals with a diameter of $100 \mathrm{~mm}$ were successfully grown at TRS Technologies [the schematic figure is given in Fig. 2(a), where the color change represents the Ti segregation]. The samples used in this study were cut along the growth direction with different PT contents, where both rhombohedral-like (low PT content) and tetragonal-like (high PT content) ternary crystals [i.e., $0.26 \mathrm{PIN}-0.47 \mathrm{PMN}-0.27 \mathrm{PT}, 0.26 \mathrm{PIN}-$ 0.44PMN-0.30PT, 0.26PIN-0.42PMN-0.32PT, and 0.26PIN-0.38PMN0.36PT crystals (shortened to PINMT27, PINMT30, PINMT32, and PINMT36, respectively)] were considered. The quantitative compositions of various samples were further confirmed by synchrotron submicron x-ray fluorescence experiments conducted at beamline 2ID-D, Advanced Phonon Source (APS), Argonne National Laboratory (ANL). Their elemental composition (such as In and Ti) was detected, and relative percentages are given in Fig. 2(b).

Domain configurations in the PIN-PMN-PT single crystal samples were determined by the polar directions of crystal symmetry [Fig. 2(c)] and the poling process. By poling the crystals along different crystallographic directions, various artificial domain configurations can be formed (for details see Table SI in the supplementary material), leading to very different piezoelectric, electromechanical, and loss properties. Thus, to fully characterize the material properties of the PIN-PMN-PT crystals, we poled the crystals along the [001] and [111] directions and studied their piezoelectric coefficients and electromechanical couplings [Figs. 2(d) and 2(e)]. As listed in Table S1, single domain configuration is established in [001] poled high-PT and [111] poled low-PT content crystals, while [001] poled low-PT and [111] poled high-PT content crystals have $4 \mathrm{R}$ and $3 \mathrm{~T}$ engineered-domain configurations, respectively. ${ }^{41}$ As shown in Figs. 2(d) and 2(e), PINPMN-PT possess comparable piezoelectric coefficients $\mathrm{d}_{33}$ and electromechanical coupling $\mathrm{k}_{33}$ to their PMN-PT binary counterpart. The ferroelectric-paraelectric phase transition temperatures observed in PIN-PMN-PT are particularly interesting, around $155^{\circ} \mathrm{C}-240{ }^{\circ} \mathrm{C}$, which are $30^{\circ} \mathrm{C}-65^{\circ} \mathrm{C}$ higher than the PMN-PT crystals with similar PT contents [Fig. 2(f)]. Together with their high coercive fields and enhanced field-induced phase transition level (see Fig. S1 in the supplementary material), the PIN-PMN-PT crystals promise a good candidate for broadened temperature usage range and drive field stability.

Several thermodynamic-based models, such as the instability of the structure phase at the MPB, ${ }^{9}$ ease of polarization rotation/extension, ${ }^{3,7}$ and critical phenomenon, ${ }^{42}$ have been proposed to understand the ultrahigh piezoelectric property of relaxor-PT crystals. However, these mechanisms fail to explain why PIN-PMN-PT exhibits comparable piezoelectricity but greatly enhanced thermal stability when compared with their binary counterpart, even though the ternary system possesses higher $\mathrm{T}_{\mathrm{c}}$ and $\mathrm{T}_{\mathrm{rt}}$. Therefore, a comprehensive investigation and comparative analysis should be conducted on binary PMN-PT and ternary PIN-PMN-PT solid solution crystals.

To explore the origin of this interesting behavior, we first consider the intrinsic (piezoelectric lattice-deformation) and extrinsic (i.e., domain wall or phase boundary motions) contributions to the material properties. $^{15,16}$ Figure 2(g) summarizes the loss behavior of the PINPMN-PT and PMN-PT crystals. The ternary PIN-PMN-PT crystals exhibit lower loss (higher mechanical quality factor $\mathrm{Q}_{33}$ values) compared to their binary counterpart for both engineered-domain and single domain configurations. It has been generally accepted that the extrinsic domain wall motion plays a crucial role in loss behavior. Thus, we reasonably believe that the lower loss in PIN-PMN-PT is attributed to the decreased extrinsic contribution. ${ }^{17}$ Meanwhile, the extrinsic contribution to the piezoelectric activity of PIN-PMN-PT crystals should be lower than that of their binary counterpart, which is confirmed by the measurements of piezoelectric coefficients with single- and multi-domain configurations (Fig. S2 in the supplementary material). These observations demonstrate that the excellent dielectric/ piezoelectric property, together with the high thermal stability in PIN-PMN-PT, should be an intrinsic characteristic.

\section{B. Polar nanoregions (PNRs) and dielectric relaxation}

For perovskite ferroelectrics, the piezoelectric coefficient $d_{33}$ can be described as: ${ }^{43}$

$$
\mathrm{d}_{33}=2 Q_{33} \mathrm{P}_{\mathrm{S}} \varepsilon_{33},
$$

where $Q_{33}$ is the electrostrictive coefficient, $P_{S}$ is the spontaneous polarization, and $\varepsilon_{33}$ is the dielectric permittivity. Relaxor-PTs have lower electrostrictive coefficients and spontaneous polarization compared to those of ferroelectric $\mathrm{Pb}(\mathrm{Zr}, \mathrm{Ti}) \mathrm{O}_{3}$ (PZT), ${ }^{2,44,45}$ yet they possess much higher piezoelectric coefficients. Therefore, we can conclude that the high piezoelectric activity is attributed to the high dielectric permittivity ( 5000-10000 for domain-engineered relaxor-PT crystals with MPB compositions). ${ }^{46}$ Such a high dielectric response in the relaxor-PT system is inherently associated with the most important characteristic in relaxor-based ferroelectrics, namely, the presence of PNRs. ${ }^{47-50}$ The contribution of PNRs to the dielectric/piezoelectric response has been quantitatively characterized to be over $50 \%$ according to the recent cryogenic experimental measurements and phasefield calculations. ${ }^{46,51}$ Therefore, it is necessary to investigate the behavior of PNRs in ternary PIN-PMN-PT crystals and explore the possible difference from their binary PMN-PT counterparts.

Figure 3(a) shows the temperature-dependent dielectric response of the unpoled crystals, from which one could observe that the ferroelectric-to-paraelectric phase transitions proceed gradually with temperature rather than sharply. Such a diffuseness characteristic is a feature of relaxor, leading to the deviation from the Curie-Weiss law, where the Burn temperatures $\left(\mathrm{T}_{\mathrm{B}}\right)$ of around $167^{\circ} \mathrm{C}-256^{\circ} \mathrm{C}$ can be derived (Fig. $\mathrm{S} 3$ in the supplementary material). Below $\mathrm{T}_{\mathrm{B}}$, the deviations from the Curie-Weiss law were observed due to the existence of PNRs. Various theoretical approaches have been developed to describe 
(a)

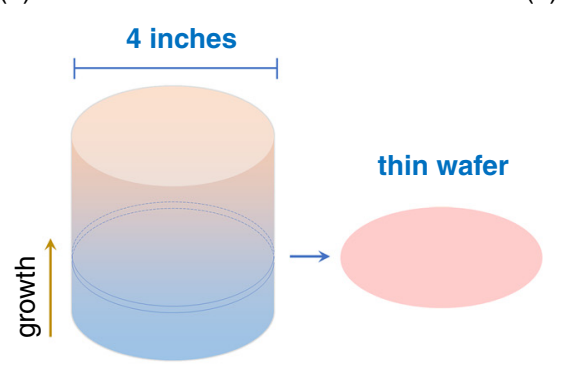

(b)

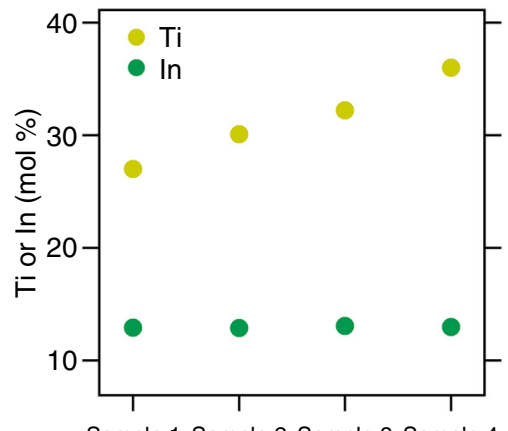

(c)

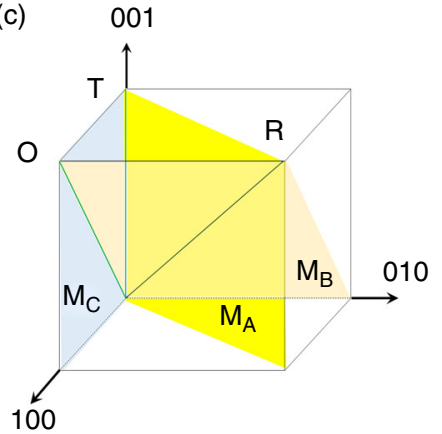

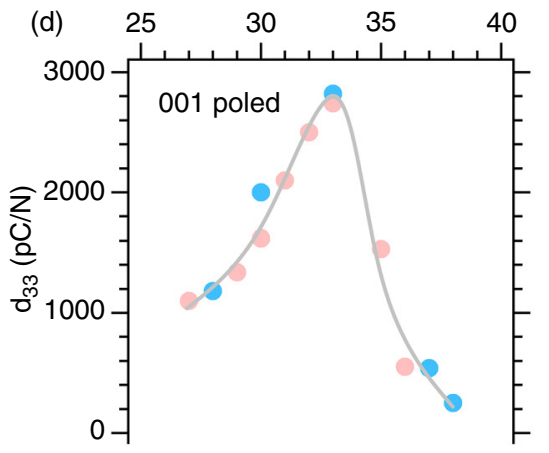

multi-domain

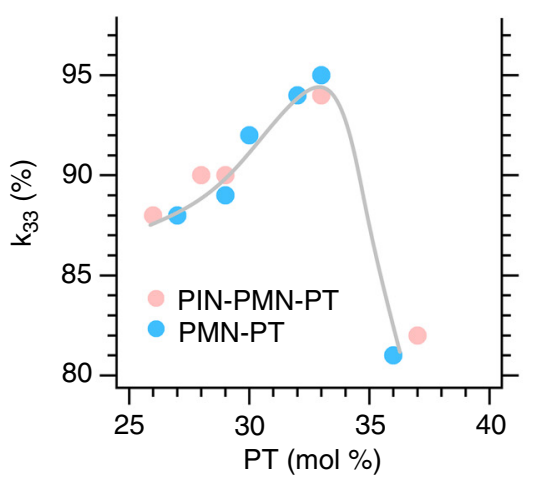

(e)

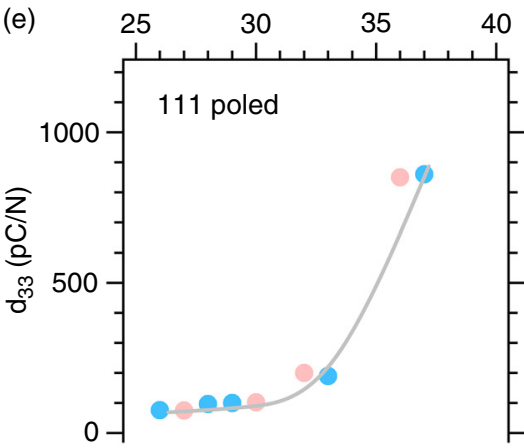

single-domain

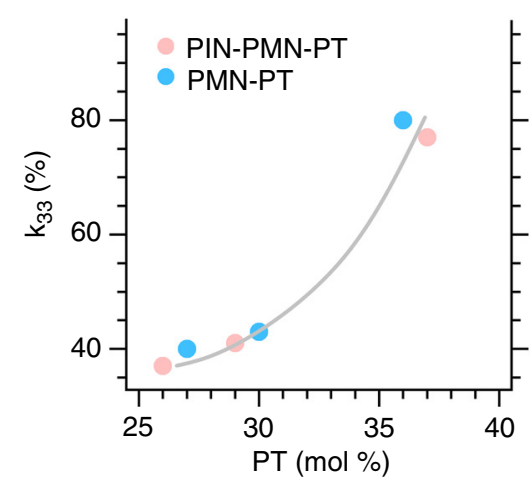

(f)
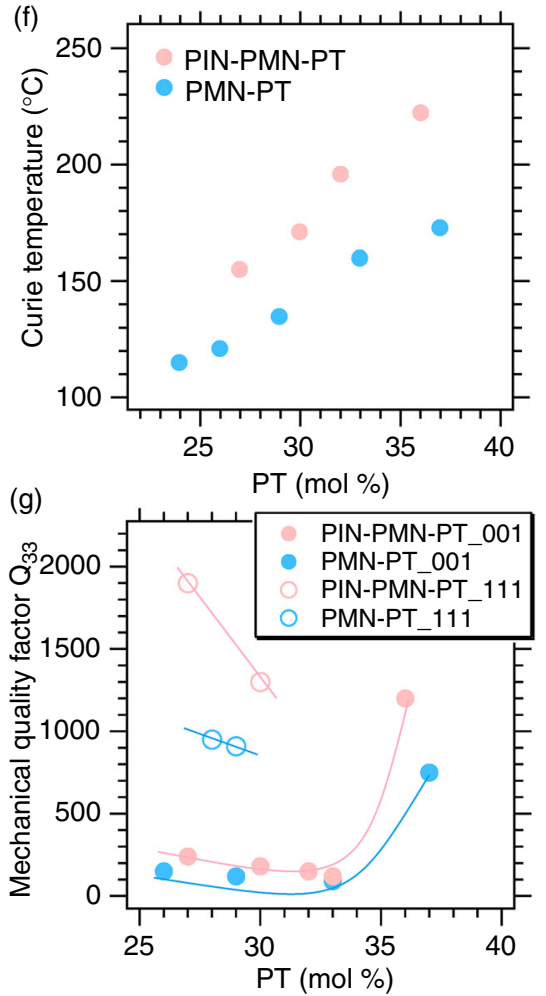

FIG. 2. Materials properties characterizations. (a) High-quality and large PIN-PMN-PT crystals (4-inches in diameter) employed in this work. Thin crystal wafers were cut for structure and materials property investigation. (b) By synchrotron-based X-ray fluorescence experiments, the quantitative compositions of four PIN-PMN-PT samples used in this work are determined as $0.26 \mathrm{PIN}-0.47 \mathrm{PMN}-0.27 \mathrm{PT}$, 0.26PIN-0.44PMN-0.30PT, 0.26PIN-0.42PMN-0.32PT, and 0.26PIN-0.38PMN-0.36PT. (c) Polarizations and mirror planes of various perovskite ferroelectric phases. ${ }^{36}$ (d) Composition dependence of piezoelectric coefficients $d_{33}$ and electromechanical coupling $k_{33}$ for PMN-PT and PINPMN-PT poled along [001 $]_{p c}$. (e) Composition dependence of piezoelectric coefficients $d_{33}$ and electromechanical coupling $k_{33}$ for PMN-PT and PIN-PMN-PT poled along $[111]_{p c}$. (f) Composition dependence of Curie temperature for PMN-PT and PIN-PMN-PT. (g) Composition dependence of mechanical quality factor for PMN-PT and PIN-PMNPT. The data shown in (d) $-(\mathrm{g})$ are from Refs. $15-17$ and $37-40$ and this work.

the relaxor behavior. ${ }^{52-55}$ The temperature-dependence of dielectric properties can be represented by a modified Curie-Weiss law as:

$$
\frac{1}{\varepsilon(T)}-\frac{1}{\varepsilon_{m}}=\frac{\left(T-T_{m}\right)^{\gamma}}{C}
$$

Here $\gamma$ and $\mathrm{C}$ are assumed to be constant, and $1 \leq \gamma \leq 2$. The parameter $\gamma$ gives information on the shape of the anomaly in paraelectric phase. When $\gamma=1$, the ideal Curie-Weiss law is observed, which is the case for normal ferroelectrics, whereas $\gamma=2$ corresponds to ideal relaxor, which has diffuse phase transition. The calculated $\gamma$ values for the studied ternary crystals are in the range of $1.47 \sim 1.65$ [Fig. 3(b)], demonstrating considerable relaxor characteristics, which is inconsistent with the nature of solid solution formed by normal-ferroelectric and relaxor end-members. The $\gamma$ parameter alone cannot represent the relaxor behavior since it only describes the slope shape of the dielectric as a function of temperature. The degree of diffuse phase transition is also related to the width of the dielectric anomaly over the same frequency range. ${ }^{56,57}$ 
(a) $\times 10^{3}$
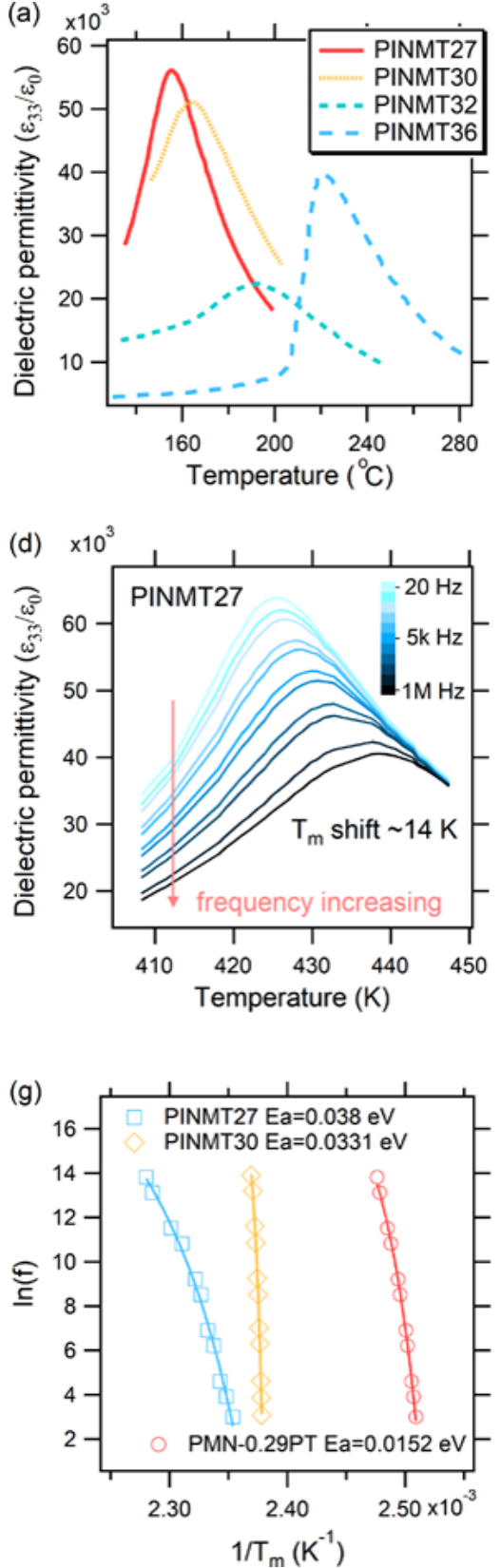

(b)

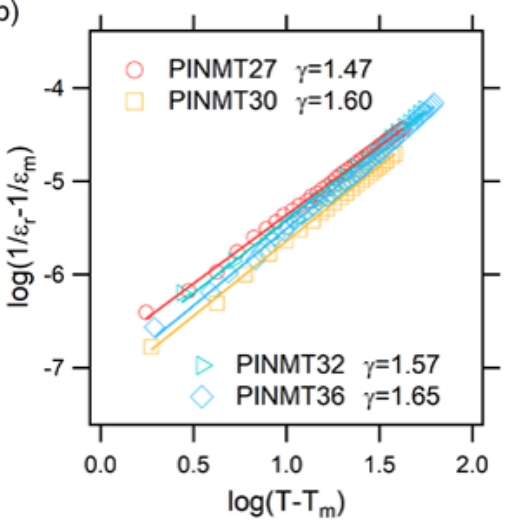

(e) $\times 10^{3}$

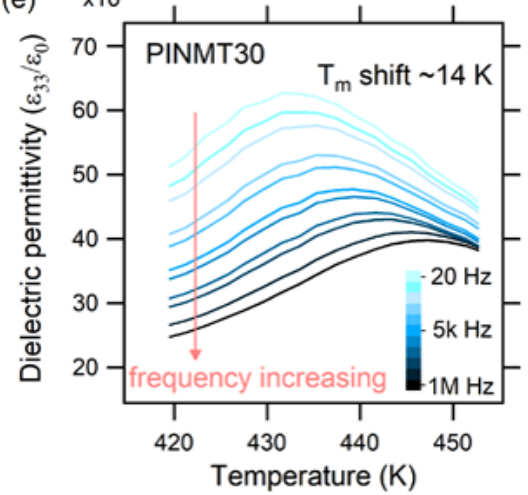

(h)

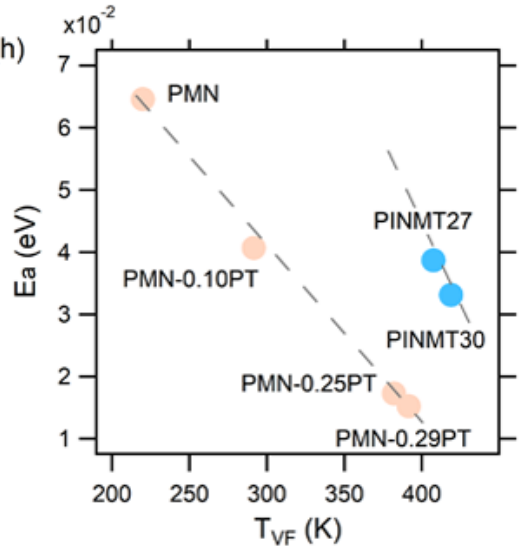

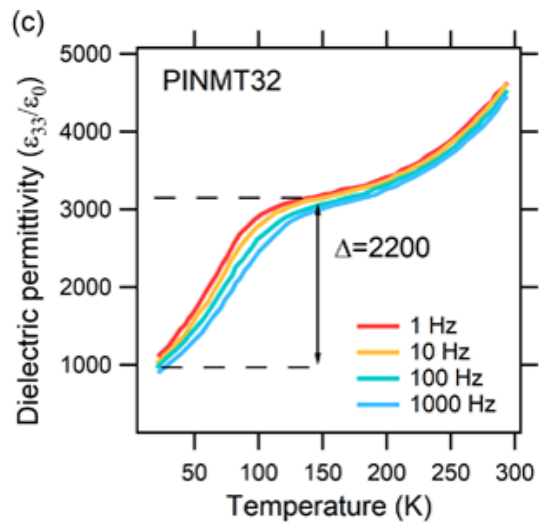

(f)

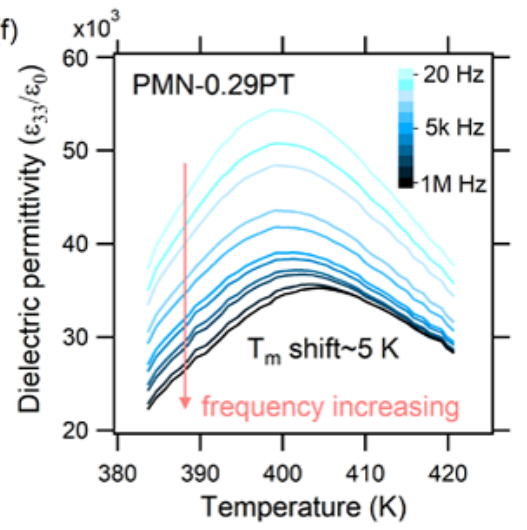

(i) 1.00 (1)

FIG. 3. Dielectric properties and relaxor behavior. (a) Temperature dependence of dielectric permittivity for unpoled PINMT27, PINMT30, PINMT32, and PINMT36 crystals, at $1 \mathrm{kHz}$. (b) $\log \left(1 / \varepsilon-1 / \varepsilon_{m}\right)$ vs $\log \left(T-T_{m}\right)$ curves at temperatures above $T_{m}$ at $1 \mathrm{kHz}$ for PINMT27, PINMT30, PINMT32, and PINMT36. Here we employed unpoled samples to obtain the relaxor behavior without any poling effects. (c) Low-temperature dielectric property for [001]-poled PINMT32 at various frequencies, from which the contribution of PNRs to the dielectric responses can be estimated. (d)-( $\mathrm{f}$ High-temperature dielectric property for unpoled PINMT27, PINMT30, and PMN-0.29PT crystals, measured at frequencies of $20,50,100,500,1 \mathrm{k}, 5 \mathrm{k}, 10 \mathrm{k}, 50 \mathrm{k}, 100 \mathrm{k}, 500 \mathrm{k}$, and $1 \mathrm{M} \mathrm{Hz}$. (g) Vogel-Fulcher fitting results on the data shown in (d)-(f), from which the activation energy $E_{\mathrm{a}}$ and static freezing temperature $\mathrm{T}_{\mathrm{VF}}$ can be obtained. (h) Summary of activation energy for various PMN, PMN-PT, and PIN-PMN-PT. Data are from refs. 59, 61, and 62, and this work. (i) $T_{\mathrm{VF}} / T_{\mathrm{m}}$ value as a function of frequency for PMN-PT and PIN-PMN-PT. Data are from Refs. 59 and 61 and this work.

Thus, the values of $\gamma$ parameter derived from Eq. (2) only suggest there are considerable relaxor characteristics in PINMT, but cannot be simply used for a quantitative analysis and comparison. Figures 3(c) and $\mathrm{S} 4$ in the supplementary material show the low-temperature dielectric property of (001)-poled PINMT32 crystal. We quantitatively estimated that the contribution of PNRs to the room-temperature dielectric response is about $50 \%$, roughly the same as those of binary crystals (50\% for PMN-0.30PT). ${ }^{46}$ 
It is clear that the dielectric and piezoelectric properties of relaxor-PT crystals are dominated by intrinsic contribution, where no obvious difference is observed in the magnitude of PNRs' contributions between PIN-PMN-PT and PMN-PT crystals. However, the PNRs themselves exhibit significantly different behavior between binary and ternary crystals, which is revealed by our following dielectric measurements. Figures $3(\mathrm{~d})-3(\mathrm{f})$ show the high-temperature dielectric properties of the studied ternary crystals, where the magnitude of the dielectric permittivity decreases while the dielectric maximum shifts to higher temperatures with increasing frequency, demonstrating a strong relaxor behavior. Of particular interest is that the ternary crystals exhibit a much higher " $\mathrm{T}_{\mathrm{m}}$ shift" (shift of the dielectric maxima temperature with frequency over the range of $20 \mathrm{~Hz}$ to $1 \mathrm{M} \mathrm{Hz}$ ) than those of the PMN-PT crystals, at around $14 \mathrm{~K}$ vs $5 \mathrm{~K}$. The experimental data shown in Figs. 3(d)-3(f) can be well fitted by the Vogel-Fulcher relationship following Eq. (3): ${ }^{58}$

$$
f=f_{0} \exp \left[-E_{a} /\left(k_{B}\left(\mathrm{~T}_{\mathrm{m}}-\mathrm{T}_{\mathrm{VF}}\right)\right],\right.
$$

where $T_{V F}$ is the static freezing temperature, which can be deemed as the permittivity maximum temperature at $0 \mathrm{~Hz} ; E_{a}$ is the activation energy; $f_{0}$ is the Debye frequency; and $\mathrm{T}_{\mathrm{m}}$ is the temperature of the permittivity maximum, which is a reflection of the inability of the PNRs to follow the drive field with increasing frequency. The VogelFulcher relationship was previously introduced to describe the magnetic relaxation in spin-glass systems and then applied to relaxor ferroelectrics to understand the polarization fluctuation. $E_{a}$ represents the activation energy of polarization fluctuation in an isolated cluster arising from the development of short-range order, and a larger $E_{a}$ suggests a stronger interaction between neighboring PNRs. ${ }^{59,60}$ The fitted results are given in Fig. $3(\mathrm{~g})$, revealing that the relaxor behavior in rhombohedral crystals is analogous to a polar-glassy system. The fitted parameters $E_{a}$ of the PIN-PMN-PT crystals are particularly significant, around $0.0387 \mathrm{eV}$ for PINMT27 and $0.0331 \mathrm{eV}$ for PINMT30 because they are much higher than that of $0.71 \mathrm{PMN}-0.29 \mathrm{PT}$ $(0.0152 \mathrm{eV})$, demonstrating a stronger PNR interaction in ternary crystals. The analysis of the $\mathrm{T}_{\mathrm{VF}}$ values derived by the Vogel-Fulcher relationship also confirms this behavior. Figure 3(h) summarizes the $E_{a}$ and $\mathrm{T}_{\mathrm{VF}}$ values for various crystals, showing that PIN-PMN-PT exhibits higher $E_{a}$ and $\mathrm{T}_{\mathrm{VF}}$ values compared to its binary counterpart with similar PT content. Figure 3(i) shows the calculated ratios of $\mathrm{T}_{\mathrm{VF}} / \mathrm{T}_{\mathrm{m}}$, which decrease with increasing frequency due to the considerable " $\mathrm{T}_{\mathrm{m}}$ shift." The magnitude of " $\mathrm{T}_{\mathrm{m}}$ shift" is associated with the interactions between the PNRs and the development of local correlations, and $\mathrm{T}_{\mathrm{VF}} / \mathrm{T}_{\mathrm{m}}$ is a semi-quantitative parameter to evaluate the interaction. Compared with binary crystals, lower $\mathrm{T}_{\mathrm{VF}} / \mathrm{T}_{\mathrm{m}}$ was observed in PINPMN-PT crystals, again confirming the strong PNR interactions, from which a higher degree of deviation from classic ferroelectric behavior can be expected.

Therefore, considering their higher $\mathrm{T}_{\mathrm{m}}$ shift, higher $E_{a}$, and lower $\mathrm{T}_{\mathrm{VF}} / \mathrm{T}_{\mathrm{m}}$ values, we can reasonably conclude that PIN-PMN-PT crystals exhibit more relaxor-like characteristics than PMN-PT crystals. This can be explained by the microstructure, where the B-site ordering in PIN-PMN-PT is weaker than PMN-PT as a result of the mixture of four heterovalent cations on the same crystallographic location. Therefore, it becomes much more difficult for PIN-PMN-PT to establish homogeneous polarization ordering but only short-range ordering between neighboring clusters. A larger amount of PNRs with smaller size will form, ${ }^{63,64}$ and it is unsurprising to observe stronger PNR interaction associated with enhanced relaxor features in ternary PINPMN-PT, which could provide information crucial to understanding the superior piezoelectricity in relaxor ferroelectrics.

\section{Phase coexistence in PIN-PMN-PT solid solutions}

It has been demonstrated that PNRs can significantly affect the structural properties of the relaxor ferroelectrics due to a strong interaction between the PNR and the propagation of acoustic phonons, from which phase instability will be induced and finally contribute to the ultrahigh piezoelectric response. ${ }^{65,66}$ Therefore, we are motivated to re-examine the microstructure of ternary PIN-PMN-PT crystals to explore their possible structural instability and hidden crystallographic symmetry, and provide a full picture of the phase diagram.

The structure of ferroelectric solid solutions with MPB composition is sensitive to many factors, including strain/stress, domain size, local cation ordering, and chemical inhomogeneity, which makes comprehending them fully challenging. ${ }^{8}$ Recent developments in synchrotron-based detection techniques have pushed the achievable spatial resolution and the sensitivity of diffraction, imaging, and spectroscopy to an unparalleled level, providing new insight into the structure-property relationship of ferroelectric solid solutions. Here, we conducted in situ synchrotron X-ray diffraction investigations at the 11BM station of Argonne National Laboratory with ultrahigh resolution and flux (Fig. S5 in the supplementary material) to comprehensively study the phase diagram of the PIN-PMN-PT system. The results help us to monitor the crucial MPB diffusion phenomenon.

The room temperature structures of the PIN-PMN-PT system are given in Figs. 4 and S6-S12 in the supplementary material. It has been reported that PINMT27 and PINMT30 possess a pure rhombohedral phase with a $R 3 m$ space group due to its low PT content. However, careful analysis of the high-resolution synchrotron XRD data shown in Figs. 4(a)-4(d) reveals a multi-peak characteristic for the pseudocubic $\{200\}_{\mathrm{pc}}$ diffraction peak within the diffraction angle of $11.68^{\circ}-11.80^{\circ}$. Thus, the obtained XRD pattern cannot be satisfied by the single $R 3 m$ space group since $R 3 m$ only allows for one $\{200\}_{\mathrm{pc}}$ peak, and there must be a low-symmetry phase contributing to the $\{200\}_{\text {pc }}$ peak splitting. As expected, the high $R_{p}$ value of $11.74 \%$ from the refinement results reveals that a single $R 3 m$ model cannot accurately describe the measured data (Fig. $\mathrm{S} 6$ in the supplementary material). Then, two mix-phase models, $R 3 m+P m\left(\mathrm{R}+\mathrm{M}_{\mathrm{C}}\right)$ and $R 3 m+C m$ $\left(\mathrm{R}+\mathrm{M}_{\mathrm{A}}\right)$, were considered. We found that the fitting quality of the refinement using $R 3 m+P m$ set [Figs. 4(a) and 4(c)] was superior to $\mathrm{R} 3 m+\mathrm{Cm}$ (Figs. S7 and S8 in the supplementary material), as confirmed by the relatively small $\mathrm{R}_{\mathrm{p}}$ (7.67\% for PINMT27 and $7.38 \%$ for PINMT30), where considerable monoclinic phase fractions of $57 \%$ and 74\% were found in PINMT27 and PINMT30, respectively. Although the raw data could be better fitted by two monoclinic phases $(P m+C m)$ due to the larger number of the refined structural parameters, we still believe that the $R 3 m+P m$ model is the most accurate set representing the real material structure of PINMT27 and PINMT30 for the following two reasons: (i) These two compositions are located at the rhombohedral-rich MPB side. Thus, the $R 3 m$ space group should not be completely ignored; (ii) $P m$ belongs to a monoclinic phase, which is essential for providing the polarization rotation path according to Landau free-energy theory. ${ }^{3,9,15-17}$ Therefore, we demonstrate that two phases with competing symmetries coexist in ternary 

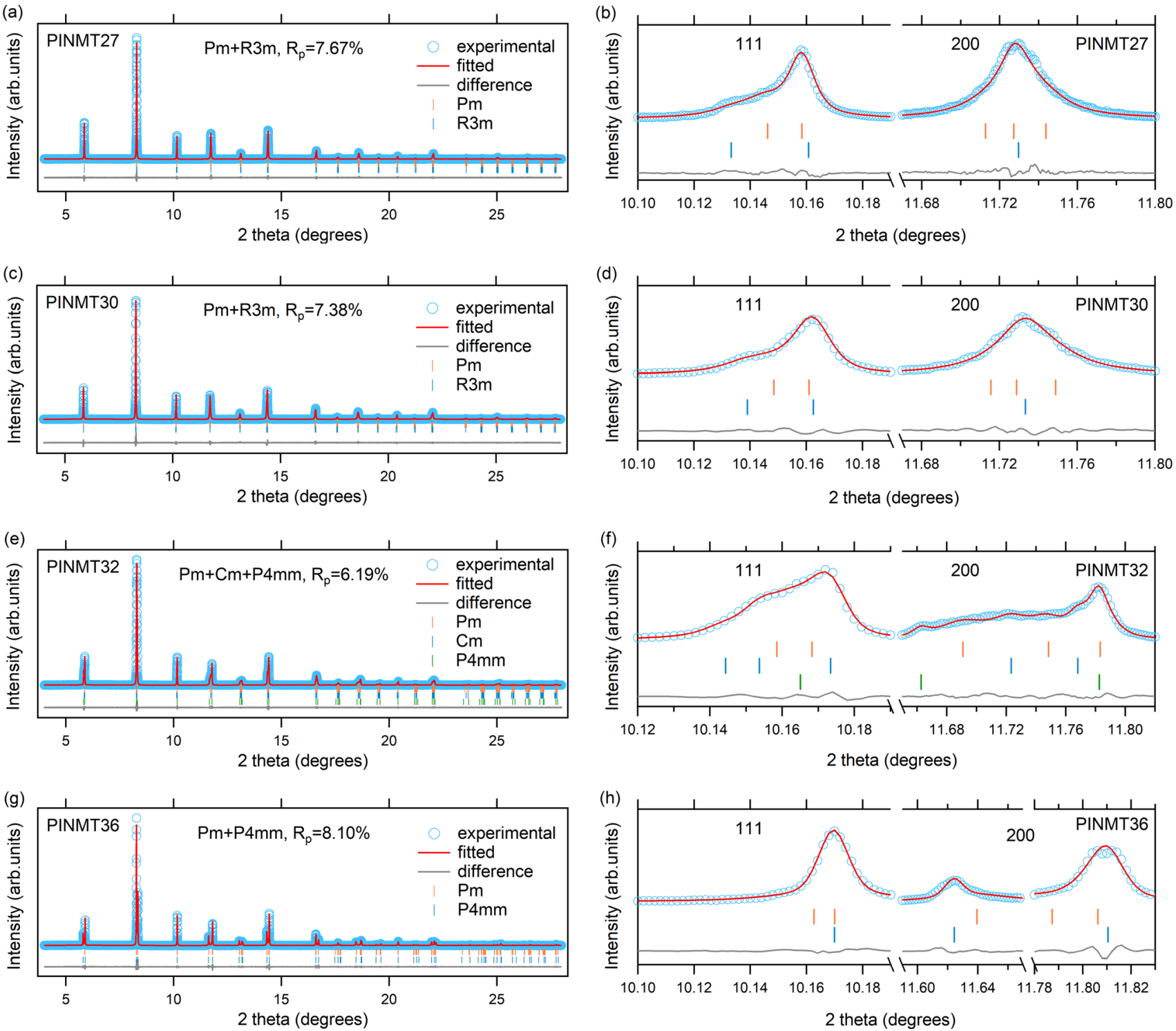

FIG. 4. High-resolution synchrotron XRD investigations on PIN-PMN-PT at room temperature, and the optimal Rietveld refinements. (a), (c), (e), and (g) give the whole patterns for PINMT27, PINMT30, PINMT32, and PINMT36, respectively. (b), (d), (f), and (h) highlight the selected reflections. The phase coexistence behavior has been well convinced for all four compositions.

PIN-PMN-PT with relative low PT content. Such a characteristic is significantly different from the binary PMN-PT system, in which the pure $R 3 m$ symmetry was confirmed in $0.72 \mathrm{PMN}-0.28 \mathrm{PT}$ by using the same synchrotron facility.

A similar analysis strategy was also employed on the high-PT composition crystal, PINMT36. Considering the fact that it is located on the tetragonal-rich MPB side, the $P 4 \mathrm{~mm}$ space group cannot be ruled out. However, a simple, pure phase model with $P 4 \mathrm{~mm}$ cannot satisfy the XRD patterns shown in Figs. 4 (g) and 4(h). P4 mm only allows a single peak of (200) and a single peak of (002), however, in Fig. $4(\mathrm{~h})$ at least two Bragg reflections are obviously identified for $(200)_{\mathrm{pc}}\left(2\right.$ theta $\left.\sim 11.81^{\circ}\right)$ and an asymmetric peak profile was found for $(002)_{\mathrm{pc}}\left(2\right.$ theta of $\left.11.60^{\circ}-11.65^{\circ}\right)$. From the Rietveld refinement [Figs. 4(h) and S12], $P m\left(\mathrm{M}_{\mathrm{C}}\right)$ is suggested to be in the secondary lowsymmetry-phase rather than $C m\left(\mathrm{M}_{\mathrm{A}}\right)$, which is similar to the condition of PINMT27 and PINMT30, and consistent with the binary PMN-PT system with MPB composition. ${ }^{35}$ For the intermediate composition PINMT32, more complicated diffraction patterns with a broader profile were observed [Figs. 4(e) and 4(f)]. From a detailed analysis of the peak positions and intensities of the fitted profiles, none of the two-phase models (such as $P 4 \mathrm{~mm}+\mathrm{Cm}, \mathrm{P} 4 \mathrm{~mm}+\mathrm{Pm}$, and even two monoclinic $P m+C m$ ) were found to fit well for the observed 
features (Figs. S9-S11 in the supplementary material). Thus, the threephase model $\mathrm{Pm}+\mathrm{Cm}+\mathrm{P} 4 \mathrm{~mm}$ offers a realistic solution [Fig. 4(f)], as supported by the significantly small $\mathrm{R}_{\mathrm{p}}$ value of $6.19 \%$.

Based on the above analysis, phase coexistence is revealed in all studied samples. Considering that the MPB of the relaxor-PT system is characterized by multi-phase features with complex phase behavior, ${ }^{34,35}$ such an expanded composition-span in PIN-PMN-PT strongly suggests a diffused MPB characteristic. It should be noted that the phase coexistence in the ternary relaxor-PT is an intrinsic feature of chemically complex systems, which is in good agreement with the observations in PMN-PT and PZT systems. ${ }^{35,67,68}$ Such a phase coexistence in relaxor-based ferroelectrics cannot be explained by the long-range compositional fluctuations since the phase stability can be disturbed by local PNRs. Note that the MPB in ferroelectrics is associated with random fields, compositional heterogeneity, polar nanoregions, mesostructural heterogeneity, and nanodomains. In this context, the diffused MPB can be regarded as a MPB with local structural heterogeneity, which is more favorable in ternary PIN-PMN-PT than binary PMN-PT. Therefore, from a microstructure viewpoint, we can also understand why MPB occurs more easily in PIN-PMN-PT.

\section{Phase diagram, improved thermal stability, and high piezoelectricity}

To further explore the properties of the ternary PIN-PMN-PT crystals with MPB compositions, we collected the XRD patterns as a function of temperature from 100 to $600 \mathrm{~K}$, and conducted the detailed fitting procedures. Figures 5(a) -5 (d) show the temperature-driven structural evolutions and phase fractions for PINMT27, PINMT30, PINMT32, and PINMT36, respectively. For PINMT27 and PINMT30, the room-temperature phase of $\mathrm{R}+\mathrm{M}_{\mathrm{C}}$ was suitable at low temperature down to $100 \mathrm{~K}$ and persisted up to $405 \mathrm{~K}$ (for PINMT27) and $375 \mathrm{~K}$ (for PINMT30), at which the two phases merged to a single $\mathrm{M}_{\mathrm{C}}$, followed by $\mathrm{M}_{\mathrm{C}} \rightarrow$ Cubic (space group: $\operatorname{Pm} \overline{3} \mathrm{~m}$ ) phase transitions occurring at 424 and $444 \mathrm{~K}$, respectively. A similar phase transition sequence (two ferroelectric phases $\rightarrow$ single ferroelectric phase $\rightarrow$ paraelectric cubic phase) was also observed in PINMT36, in which $\mathrm{T}+\mathrm{M}_{\mathrm{C}}$ merged to $\mathrm{T}$ at $483 \mathrm{~K}$ and finally transformed to a cubic phase at $496 \mathrm{~K}$. More complicated ferroelectric phase transitions upon heating PINMT32 were further resolved, which followed $\mathrm{M}_{\mathrm{A}}+\mathrm{M}_{\mathrm{C}}+\mathrm{T}$ to $\mathrm{M}_{\mathrm{C}}+\mathrm{T}$, and finally to the cubic phase. Detailed temperaturedependent structural parameters of all studied samples are summarized in Figs. S13-S20 in the supplementary material.

According to the results of the phase evolutions shown in Figs. 5(a)-5(d), we re-plotted the composition-temperature phase diagram of the PIN-PMN-PT ternary system. The various monoclinic phases clearly diffuse into the $\mathrm{R}$ and $\mathrm{T}$ regions. Consequently, as shown in Fig. 5(e), MPB is not a narrow compositional region containing lowsymmetry phase(s) (3\% 5\% for PMN-PT; ${ }^{35,69} 2 \%$ for PZN-PT $\left.{ }^{34}\right)$, but an extremely broad MPB region covering at least $9 \%$ PT contents, namely, a "diffused MPB." Here, the term "diffused" means lowsymmetry monoclinic phase significantly expands into the $\mathrm{R}$ and $\mathrm{T}$ phase regions, being distinct from the $\mathrm{Pb}\left(\mathrm{Zr}_{0.52} \mathrm{Ti}_{0.48}\right) \mathrm{O}_{3}-\mathrm{BaTiO}_{3}$, $\mathrm{Pb}\left(\mathrm{Zn}_{1 / 3} \mathrm{Nb}_{2 / 3}\right) \mathrm{O}_{3}-\mathrm{Pb}\left(\mathrm{Zr}_{1 / 2} \mathrm{Ti}_{1 / 2}\right) \mathrm{O}_{3}$, and $\mathrm{Bi}\left(\mathrm{Mg}_{3 / 4} \mathrm{~W}_{1 / 4}\right) \mathrm{O}_{3}-\mathrm{PbTiO}_{3}$ systems, in which there is no low-symmetry phase observed and/or only narrow MPBs were reported. ${ }^{70-74}$ Such a broad composition region with co-existing phases indicates a strong competition among various ferroelectric phases with different polarization vectors.
The diffusion of monoclinic symmetry in PIN-PMN-PT can also be understood from a system energy perspective and confirmed by implementing first-principle calculations with long-range force considerations. We employed $0.26 \mathrm{PIN}-0.49 \mathrm{PMN}-0.25 \mathrm{PT}$ and $0.75 \mathrm{PMN}-$ $0.25 \mathrm{PT}$ as an example pair to compare the energy of the low-symmetry phase and parent ferroelectric phase. As shown in Fig. 5(f), the enthalpy per unit volume of $R 3 \mathrm{~m}$ is obviously lower than that of $P \mathrm{~m}$ in PMN-PT, while for PIN-PMN-PT, the enthalpy values of two symmetries are similar and the energy of $P m$ is even lower, which agrees well with our experimental results.

PIN-PMN-PT has a higher Curie temperature than its binary PMN-PT counterpart, and better thermal stability of structure/functionality can be expected, which can be explored from the aspect of the Landau-Ginzburg-Devonshire (LGD) theory and temperature-driven variation of the order parameters. From LGD theory on phase transition, the free energy of the system can be expanded in a power series of the order parameter $\varphi:^{7}$

$$
F=F_{0}+\sum_{n} \frac{1}{n} \alpha_{n} \varphi^{n} .
$$

Here, $\varphi$ is a nonzero value below a critical point and zero for the highsymmetry phase. For the monoclinic phase, $\beta-90^{\circ}(\beta$ is the monoclinic angle, namely, the angle between lattice $a$ and lattice $c$ ) can be used as order parameters, allowing the free energy to be expanded. Figure $5(\mathrm{~g})$ compares the temperature-driven evolutions of $\beta-90^{\circ}$ in the PINPMN-PT and PMN-PT systems with different monoclinic phases. A more drastic change in order parameter as a function of temperature was observed in PMN-PT compared to PIN-PMN-PT. Thus, PINPMN-PT exhibits improved phase thermal stability. Consequently, the variation in the free energy profile of PIN-PMN-PT as a function of temperature should be much less than those of the PMN-PT systems. Considering the fact that the properties in perovskite ferroelectrics are strongly associated with the polarization variation and flatness of the potential wells described by their free-energy profile, high thermal stability of functionality can be expected in a PIN-PMN-PT system.

Figures 5(h) and 5(i) show the temperature dependence of variations in lateral electromechanical coupling factors $\mathrm{k}_{31}$ and piezoelectric coefficients $d_{31}$ for PIN-PMN-PT and PMN-PT crystals, respectively. We observed that $\mathrm{k}_{31}$ maintained the same values with increasing temperature for PIN-PMN-PT up to $400 \mathrm{~K}$, above which it decreased sharply due to the partial depolarization, ${ }^{76}$ which is $30 \mathrm{~K}$ higher than PMN-PT. Meanwhile the piezoelectric coefficients $\mathrm{d}_{31}$ of PIN-PMN-PT and PMN-PT were found to increase with increasing temperature and reach maxima at respective depolarization temperatures, above which they decrease significantly. It is noteworthy that PIN-PMN-PT has greater thermal stability in $\mathrm{d}_{31}$, which is evidenced by the slow increasing slope and higher depolarization temperature. As shown in Fig. 5(i), for PIN-PMN-PT crystals, the piezoelectric variation is $\sim 70 \%-90 \%$ over the temperature range of $273-373 \mathrm{~K}$, much less compared to its binary counterpart $\mathrm{Pb}\left(\mathrm{Mg}_{1 / 3} \mathrm{Nb}_{2 / 3}\right) \mathrm{O}_{3}-\mathrm{PbTiO}_{3}$ where large piezoelectric variation, $\sim 200 \%$, is evidenced over the same temperature range.

Of particular importance is that the improved thermal stability in the ternary PIN-PMN-PT system is not at the cost of decreased piezoelectric properties, from which a mechanism on the diffused MPB is proposed and shown schematically in Fig. 6. On the one hand, due to the presence of low-symmetry phase over a broader composition range in PIN-PMN-PT, the free energy profile becomes flatter than 
(a)
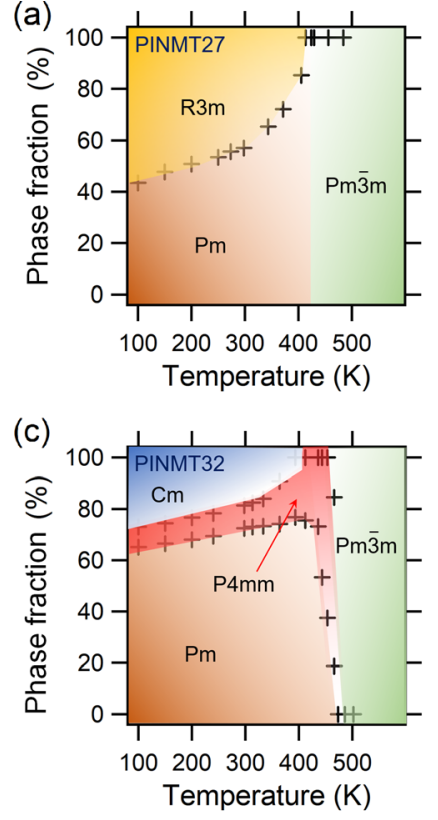
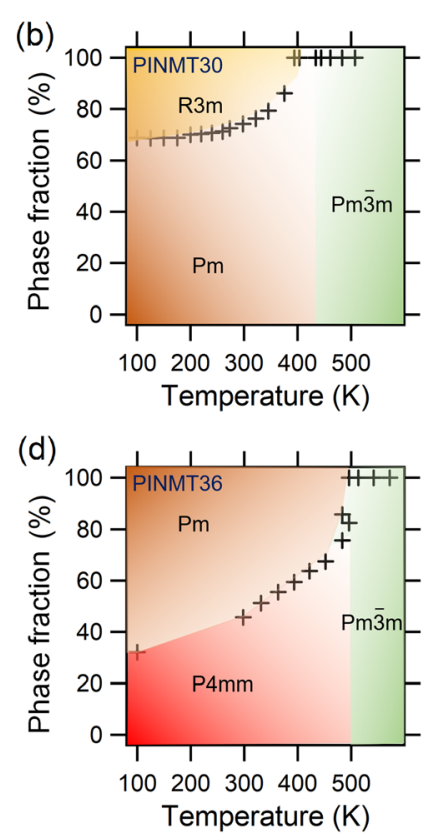

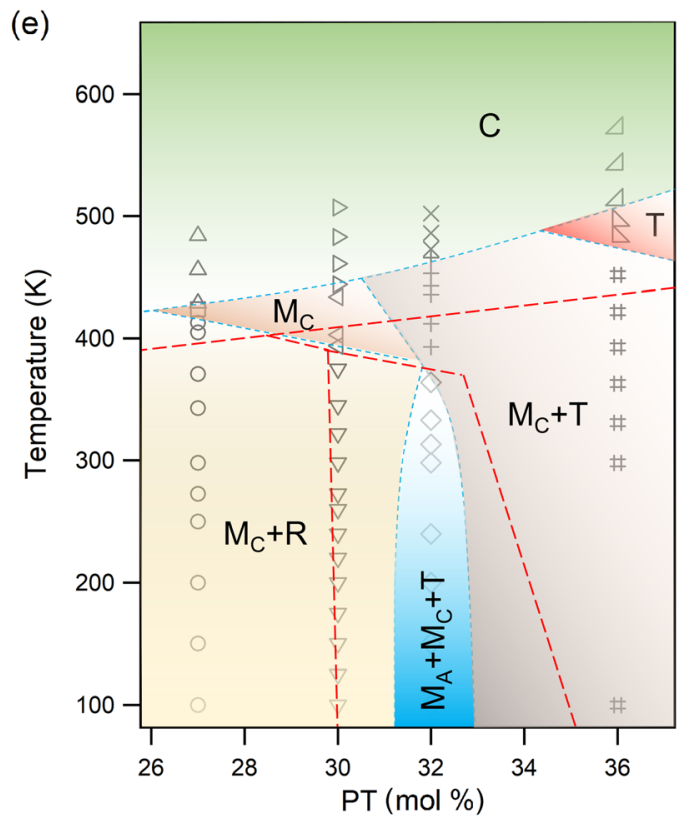

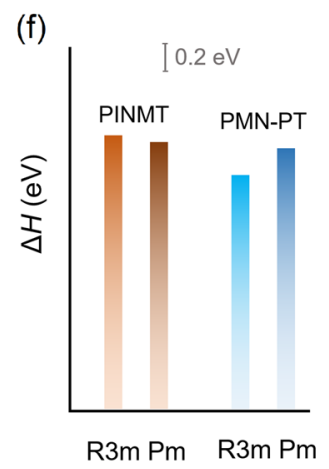
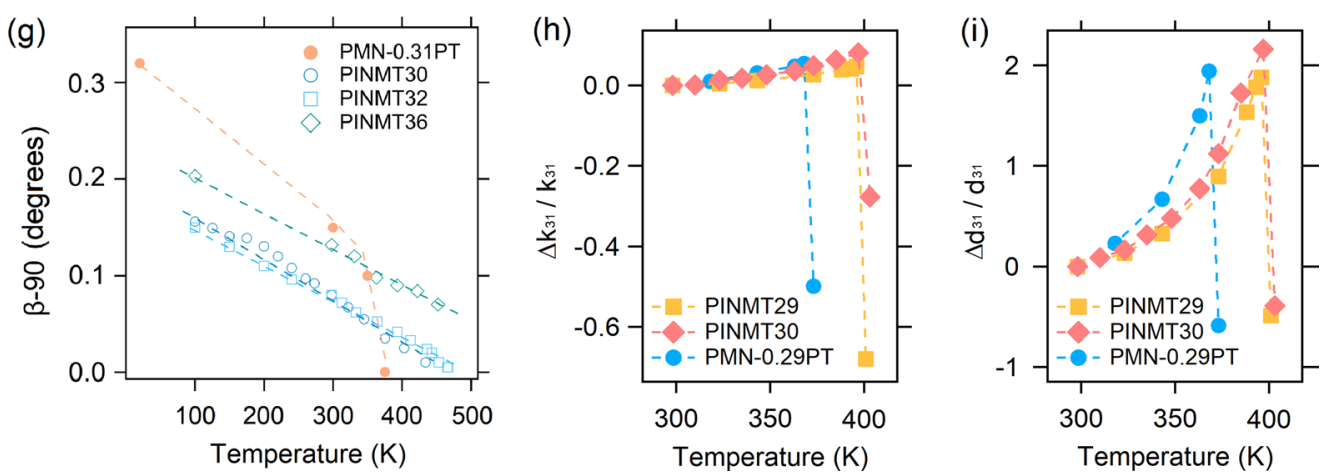

FIG. 5. Diffused morphotropic phase boundaries in the ternary PIN-PMN-PT system. (a)-(d) Temperature dependence of phase fractions for PINMT27, PINMT30, PINMT32, and PINMT36. (e) Temperature-composition phase diagram of $0.26 \mathrm{PIN}-(0.74-x) \mathrm{PMN}-\mathrm{XPT}$. The markers are directly from our experiments. The dashed red lines show the MPB region of the binary PMN-PT system, in which the concentration range of $\mathrm{PT}$ is only $2 \%-3 \%$ at room temperature. ${ }^{17}$ (f) Calculation results of enthalpy per unit cell for PMN$0.25 \mathrm{PT}$ and 0.26PIN-0.49PMN-0.25PT with $R 3 \mathrm{~m}$ and $\mathrm{Pm}$ symmetries. (g) The temperature dependence of order parameter $\beta$-90 in the monoclinic Pm phase for various relaxor-PT. For PMN-0.31PT, the data are from Ref. 35. (h) Temperature dependence of variations in $\mathrm{k}_{31}$ for (001)-poled PMN-PT and PIN-PMN-PT crystals. (i) Temperature dependence of variations in $d_{31}$ for (001)-poled PMN-PT and PIN-PMN-PT crystals. In (h) and (i) the data are from Ref. 76 and this work.

PMN-PT, yielding easier dipole variations such as polarization rotation/elongation under external electric field, where the competition between co-existing ferroelectric phases is responsible for the ultrahigh piezoelectricity [Fig. 6(a)]. On the other hand, considering the higher Curie temperature of PIN-PMN-PT, it has a more stable thermal status than PMN-PT at room temperature [Fig. 6(b)], accounting for the inferior piezoelectric activity but improved thermal stability, which generally occurs in most ferroelectric solid solution system as suggested in Fig. 1.

As shown in Fig. 5(g), for an individual phase in PIN-PMN-PT, the improved thermal stability of phase can be confirmed by temperature dependence of order parameter, nevertheless the energy gap between various ferroelectric phases at a given temperature is smaller than PMN-PT as seen in Figs. 5(a)-5(f). Therefore, considering these two opposite effects, PIN-PMN-PT exhibit the net result of improved thermal stability while possessing almost the same room temperature piezoelectric activity as PMN-PT, as schematically depicted in Fig. 6(c). At room temperature, PIN-PMN-PT is farther away from temperature-driven phase boundaries than PMN-PT, whereas has a diffused MPB characteristic. The former factor dominates improved thermal stability, and the latter make considerable contribution to piezoelectric activity.

\section{CONCLUSION}

We report the phase coexistence and the diffusion of the lowsymmetry phase in ternary PIN-PMN-PT solid solutions, which are 


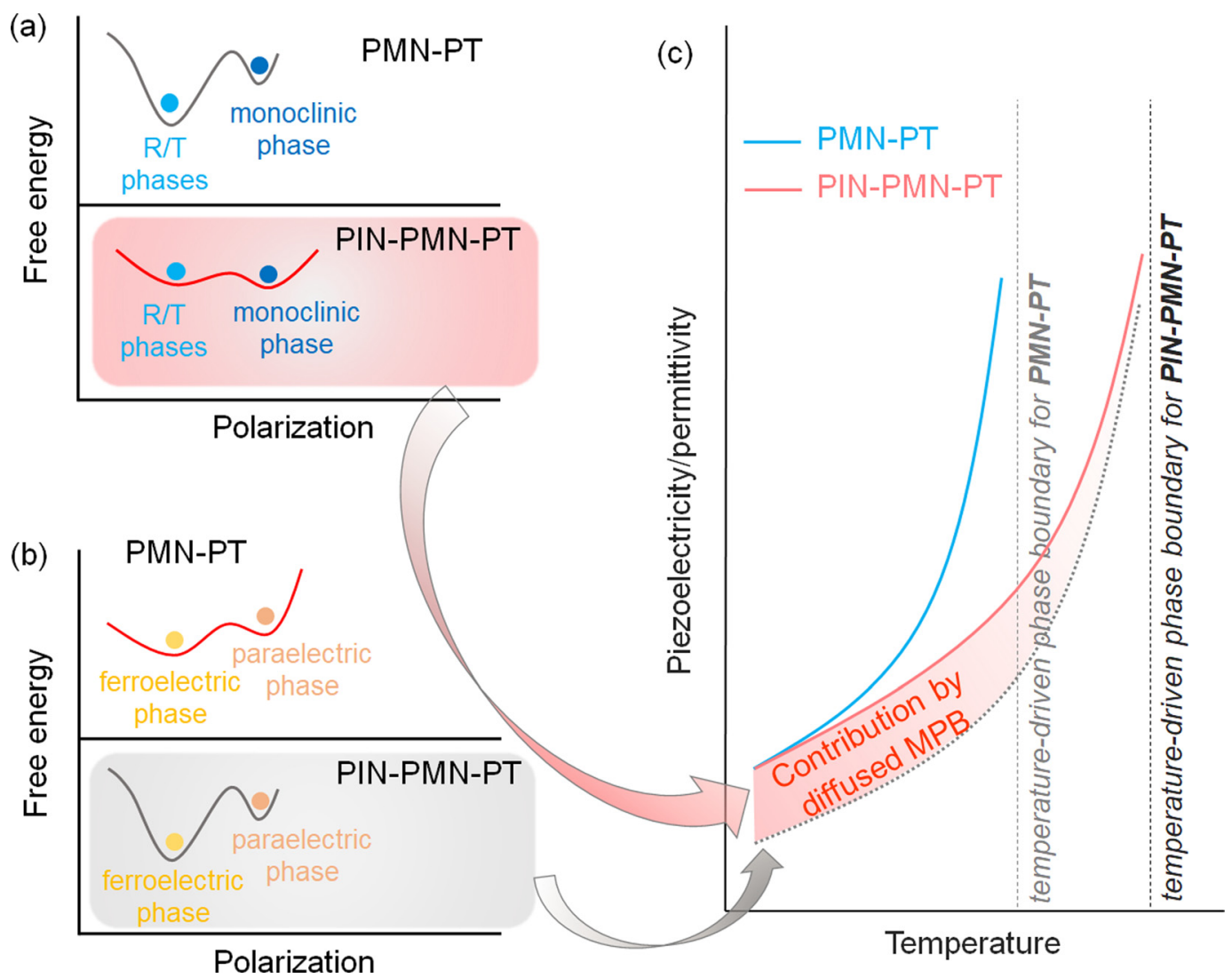

FIG. 6. Schematic mechanism of high piezoelectricity with improved thermal stability in PIN-PMN-PT. (a) and (b) Comparisons of free-energy profiles between PMN-PT and PIN-PMN-PT, in the aspect of composition-driven and temperature-driven phase transitions, respectively. (c) The mechanism of high piezoelectricity with improved thermal stability: as shown in Figs. $5(\mathrm{~g})$ and $6(\mathrm{~b})$, for an individual phase in PIN-PMN-PT, the improved thermal stability of phase is confirmed by temperature dependence of order, and improved thermal stability (farer away from temperature-driven phase boundaries) can be understood, resulting in the inferior piezoelectric activity (grey dashed line). However, a diffused MPB characteristic leads to easier dipole variations such as polarization rotation/elongation under external electric field, where the competition between co-existing ferroelectric phases is responsible for the ultrahigh piezoelectricity [Fig. 6(a)]. Thus, the net result is that PIN-PMN-PT exhibits almost the same piezoelectric response as PMN-PT at room temperature (blue and red lines for PMN-PT and PIN-PMN-PT, respectively), meanwhile has improved thermal stability. The compensated piezoelectricity contributed by diffused MPB is highlighted by the pink area. Considering the fact that the piezoelectric property is strongly associated with the composition in relaxor-PT solid solutions, in the discussion of Fig. 6, the PMN-PT and PIN-PMN-PT have the same PT contents, where the comparable room temperature piezoelectric coefficients can be observed.

responsible for the comparably high dielectric/piezoelectric properties compared to their binary PMN-PT counterparts, while possessing greatly improved thermal stability. The emergence of strong relaxorlike behavior results in intense interaction between the PNRs and acoustic waves, accounting for symmetry breaking. We also re-plotted the composition-temperature phase diagram of PIN-PMN-PT, which is crucial for materials engineering of this complicated system. These results inspire us to induce additional structural heterogeneity for expanding the phase coexistence region to achieve high piezoelectricity, meanwhile the endmember with elevated Curie temperature in the solid solution system is expected to play a positive role in the property thermal stability. The impacts of the enhanced heterogeneity and Curie temperature on piezoelectricity will counterpoise each other, leading to minor piezoelectric variations, but with improved thermal stability. The diffused MPB with a broad composition range consisting of low-symmetry phase leads to high-performance materials with high temperature/field stability, giving solid proof that MPB can be broadened to a highly expansive composition-temperature region in solid solution ferroelectrics. A similar phenomenon could be extended to other ferroic systems such as ferromagnetic and multiferroic materials.

\section{EXPERIMENTAL SECTION}

\section{A. Crystal growth and sample preparation}

The PIN-PMN-PT crystals were grown by a modified Bridgman method at TRS Technologies (State College, PA, USA). Real-time Laue $\mathrm{x}$-ray back diffractions with an accuracy of $0.5^{\circ}$ were employed to determine the orientations of the samples. The dimensions and aspect ratios of the piezoelectric resonator samples were specified by the IEEE standards of piezoelectricity. ${ }^{77}$ Vacuumsputtered gold was applied to the faces of the samples as electrodes. The samples were poled along different crystallographic directions at room temperature with an electric field of $10-20 \mathrm{kV} \mathrm{cm}^{-1}$ to form 
stable engineered-domain configurations or at elevated temperatures under an electric field of $2-4 \mathrm{kV} \mathrm{cm}^{-1}$ by field-cooling poling method to obtain single domain states.

\section{B. Dielectric, piezoelectric, and electromechanical measurements}

The temperature dependence of the dielectric permittivity was measured using an LCR meter (HP4980) connected to a computercontrolled temperature chamber. A home-made temperature controller and Model 325 Cryogenic Temperature Controller (Lake Shore Cryotronics) were employed for high- and low-temperature tests, respectively. The piezoelectric coefficients were determined by the resonance method, following the IEEE standard on piezoelectricity. ${ }^{77}$ The electric-field-induced strain was determined using a linear variable differential transducer driven by a lock-in amplifier (Stanford Research System, Model SR830). The resonance and anti-resonance frequencies of the resonator were measured by an HP4294A impedance-phase gain analyzer. Then, the values of electromechanical coupling factors were derived, and mechanical quality factors were obtained using the 3-dB method.

\section{Synchrotron-based structure characterizations}

Before conducting structural characterizations, the samples were annealed at $800^{\circ} \mathrm{C}$ for 24 hours and then slowly cooled down to room temperature to remove the residual stress in the lattice. Synchrotron sub-micron x-ray fluorescence measurements were conducted at beamline 2ID-D, Advanced Phonon Source (APS), Argonne National Laboratory (ANL), with source energy of $20 \mathrm{keV}$ with a beam size of $0.2 \mu \mathrm{m}(\mathrm{H}) \times 0.2 \mu \mathrm{m}(\mathrm{V})$. Then, the quantitative compositions of various samples were determined by elemental analysis. Synchrotron $\mathrm{x}$-ray diffraction experiments were then performed at beamline 11BM, APS, ANL, from 100 to $600 \mathrm{~K}$. The wavelength is $0.414212 \AA$, and the instrument resolution $(\Delta d / d)$ was 0.00017 , representing a state-of-the-art $d$-spacing resolution for diffraction measurements worldwide. ${ }^{78}$ Rietveld refinements of XRD data were performed using the General Structure Analysis Structure program.

\section{First-principles simulation}

We implemented a first-principle calculation to compare the phase energies of PMN-PT and PIN-PMN-PT over their compositional diagrams. The calculations were performed under the framework of density functional theory by the Vienna $a b$ initio simulation package. ${ }^{80,81}$ The electron-ion interactions of all atoms were approximated by a projector augmented wave scheme. ${ }^{82}$ The exchangecorrelation functional was described by the generalized gradient approximation in the Perdew-Burke-Ernzerhof parameterization optimized for bulk solids. ${ }^{83,84}$ We also corrected the term of van der Waals force through a DF2 method and calculated long distance interactions up to $60 \AA .85$ Our calculation employed a $2 \times 2 \times 6$ supercell with a total number of 24 available cations on the $\mathrm{B}$ sites of $\mathrm{ABO}_{3}$ perovskite. For the specific composition of $0.75 \mathrm{PMN}-0.25 \mathrm{PT}$ and the indium incorporated $0.26 \mathrm{PIN}-0.49 \mathrm{PMN}-0.25 \mathrm{PT}$, the numbers of B-site cations are approximated in Table S2 in the supplementary material. The cell variables, volume, and atomic positions were allowed to relax to optimize interatomic forces below $0.01 \mathrm{eV} / \mathrm{A}$. Since the unit cell of all three phases ( $P 4 \mathrm{~mm}, \mathrm{Pm}$, and $\mathrm{R} 3 \mathrm{~m}$ space groups) can be regarded as minor distortion on a cubic system, we simplified to treat the three cell edges throughout our simulation equally. Another challenge is the mixing of up to four types of atoms in an affordable supercell. Here, the rule of thumb in building the supercell is to align all types of atoms to maintain the highest symmetry operations. Two types of alignments were considered: A-type with $\mathrm{Ti} /(\mathrm{Nb}, \mathrm{Mg})$ atoms alternatively forming along the $c$-axis and $\mathrm{G}$-type with $\mathrm{Ti} /(\mathrm{Nb}, \mathrm{Mg})$ aligned along the (110) plane. Our calculation showed that the A-type ordering yields lower total energy. For the indium-incorporated system, 3 indium atoms replaced 1 niobium and 2 magnesium atoms, and we tried various combinations. The structure with the lowest energy was used for data analysis. The supercells used for PMN-0.25PT and PIN-PMN-0.25PT in the simulation are given in Figs. S21 and S22.

\section{SUPPLEMENTARY MATERIAL}

See supplementary material for detailed characterizations on structure and property of various relaxor-PT crystals (Figs. S1-S22, Tables S1-S2).

\section{ACKNOWLEDGMENTS}

Gang Liu would like to acknowledge the support from NSAF (Grant No. U1930401). Qingyang Hu is supported by NSFC (Grant No. 17N1051-0213). Structure characterizations were performed at beamline 2-ID-D and beamline 11-BM at APS, Argonne National Laboratory (ANL). The use of APS facilities was supported by the U.S. Department of Energy, Office of Science, Office of Basic Energy Sciences (DEAC02-06CH11357). We thank Dr. Wesley Hackenberger and Dr. Jun Luo from TRS Technologies (State College, PA) for providing high-quality relaxor-PT single crystals. We especially thank Dr. Ho-kwang Mao for useful discussions. We appreciate Ms. Freyja O’Toole for her language assistance.

\section{REFERENCES}

1B. Jaffe, R. S. Roth, and S. Marzullo, J. Appl. Phys 25, 809 (1954).

${ }^{2}$ S. E. Park and T. R. Shrout, J. Appl. Phys 82, 1804 (1997).

${ }^{3}$ H. Fu and R. E. Cohen, Nature 403, 281 (2000).

${ }^{4}$ M. Ahart, M. Somayazulu, R. E. Cohen, P. Ganesh, P. Dera, H-k Mao, R. J. Hemley, Y. Ren, P. Liermann, and Z. G. Wu, Nature 451, 545 (2008).

${ }^{5}$ B. Jaffe, W. R. Cook, and H. Jaffe, Piezoelectric Ceramics (Academic, London, 1971), p. 136

${ }^{6}$ B. Noheda, D. E. Cox, G. Shirane, J. A. Gonzalo, L. E. Cross, and S.-E. Park, Appl. Phys. Lett. 74, 2059 (1999).

${ }^{7}$ B. Noheda, Curr. Opin. Solid State Mater. Sci 6, 27 (2002).

${ }^{8}$ B. Noheda and D. E. Cox, Phase Transitions 79, 5 (2006).

${ }^{9}$ D. Damjanovic, J. Am. Ceram. Soc 88, 2663 (2005).

${ }^{10}$ S. Yang, H. Bao, C. Zhou, Y. Wang, X. Ren, Y. Matsushita, Y. Katsuya, M. Tanaka, K. Kobayashi, X. Song, and J. Gao, Phys. Rev. Lett. 104, 197201 (2010).

${ }^{11}$ D. Kan, L. Pálová, V. Anbusathaiah, C. J. Cheng, S. Fujino, V. Nagarajan, K. M. Rabe, and I. Takeuchi, Adv. Funct. Mater. 20, 1108 (2010).

${ }^{12}$ M. Akiyama, T. Kamohara, K. Kano, A. Teshigahara, Y. Takeuchi, and N. Kawahara, Adv. Mater. 21, 593 (2009).

${ }^{13}$ W. F. Liu and X. B. Ren, Phys. Rev. Lett. 103, 257602 (2009).

${ }^{14}$ D. Damjanovic, IEEE T. Ultrason. Ferro. 56, 1574 (2009).

${ }^{15}$ S. J. Zhang and F. Li, J. Appl. Phys 111, 031301 (2012).

${ }^{16}$ E. W. Sun and W. W. Cao, Prog. Mater. Sci 65, 124 (2014).

${ }^{17}$ G. Liu, S. J. Zhang, W. H. Jiang, and W. W. Cao, Mater. Sci. Eng. R: Rep. 89, 1 (2015).

${ }^{18}$ F. Li, D. Lin, Z. Chen, Z. Cheng, J. Wang, C. Li, X. Xu, Q. Huang, X. Liao, L. Q. Chen, T. R. Shrout, and S. J. Zhang, Nat. Mater 17, 349 (2018).

${ }^{19}$ G. A. Smolenskii and A. I. Agranovskii, Sov. Phys. Solid State 1, 1429 (1960). 
${ }^{20}$ Datasheet of PI Ceramic GmbH on piezoelectric materials, 2020.

${ }^{21}$ D. Luff, R. Lane, K. R. Brown, and H. J. Marshallsay, Trans. J. Brit. Ceram. Soc. 73, 251 (1974).

${ }^{22}$ G. Robert, M. Demartin, and D. Damjanovic, J. Am. Ceram. Soc 81, 749 (2005).

${ }^{23}$ N. Vittayakorn, G. Rujijanagul, X. Tan, M. A. Marquardt, and D. P. Cann, J. Appl. Phys 96, 5103 (2004).

${ }^{24}$ V. A. Isupov, Solid State Commun 17, 1331 (1975).

${ }^{25}$ K. Kakegawa, J. Mohri, T. Takahashi, H. Yamamura, and S. Shirasaki, Solid State Commun 24, 769 (1977).

${ }^{26}$ S. K. Mishra, D. Pandey, and A. P. Singh, Appl. Phys. Lett. 69, 1707 (1996).

${ }^{27}$ W. Cao and L. E. Cross, Phys. Rev. B 47, 4825 (1993).

${ }^{28}$ S. Nomura, H. Arima, and F. Kojima, Jpn. J. Appl. Phys. 12, 531 (1973).

${ }^{29}$ J. Kuwata, K. Uchino, and S. Nomura, Ferroelectrics 37, 579 (1981).

${ }^{30}$ J. Kuwata, K. Uchino, and S. Nomura, Jpn. J. Appl. Phys. 21, 1298 (1982).

${ }^{31}$ T. R. Shrout, Z. P. Chang, N. Kim, and S. Markgraf, Ferroelectr., Lett. Sect 12, 63 (1990).

${ }^{32}$ Z.-G. Ye, P. Tissot, and H. Schmid, Mater. Res. Bull 25, 739 (1990).

${ }^{33}$ F. Li, M. J. Cabral, B. Xu, Z. Cheng, E. C. Dickey, J. M. LeBeau, J. Wang, J. Luo, S. Taylor, W. Hackenberger, L. Bellaiche, Z. Xu, L.-Q. Chen, T. R. Shrout, and S. J. Zhang, Science 364, 264 (2019).

${ }^{34}$ D. La-Orauttapong, B. Noheda, Z. G. Ye, P. M. Gehring, J. Toulouse, D. E. Cox, and G. Shirane, Phys. Rev. B 65, 144101 (2002).

${ }^{35}$ B. Noheda, D. E. Cox, G. Shirane, J. Gao, and Z. G. Ye, Phys. Rev. B 66, 054104 (2002).

${ }^{36}$ M. Davis, D. Damjanovic, and N. Setter, Phys. Rev. B 73, 014115 (2006).

${ }^{37}$ G. Liu, W. Jiang, J. Zhu, and W. W. Cao, Appl. Phys. Lett. 99, 162901 (2011).

${ }^{38}$ J. Peng, H. Luo, T. He, H. Xu, and D. Lin, Mater. Lett 59, 640 (2005).

${ }^{39}$ H. Cao and H. S. Luo, Ferroelectrics 274, 309 (2002).

${ }^{40}$ X. Z. Liu, S. J. Zhang, J. Luo, T. R. Shrout, and W. W. Cao, J. Appl. Phys 106, 074112 (2009).

${ }^{41}$ M. Davis, D. Damjanovic, D. Hayem, and N. Setter, J. Appl. Phys 98, 014102 (2005).

${ }^{42}$ Z. Kutnjak, J. Petzelt, and R. Blinc, Nature 441, 956 (2006).

${ }^{43}$ M. E. Lines and A. M. Glass, Principles and Applications of Ferroelectrics and Related Materials (Oxford University Press, Oxford, UK, 1997).

${ }^{44}$ F. Li, L. Jin, Z. Xu, D. W. Wang, and S. J. Zhang, Appl. Phys. Lett. 102, 152910 (2013).

${ }^{45}$ F. Li, L. Jin, Z. Xu, and S. J. Zhang, Appl. Phys. Rev 1, 011103 (2014).

${ }^{46}$ F. Li, S. J. Zhang, Z. Xu, and L. Q. Chen, Adv. Funct. Mater. 27, 1700310 (2017).

${ }^{47}$ L. E. Cross, Ferroelectrics 76, 241 (1987).

${ }^{48}$ M. E. Manley, D. L. Abernathy, R. Sahul, D. E. Parshall, J. W. Lynn, A. D. Christianson, P. J. Stonaha, E. D. Specht, and J. D. Budai, Sci. Adv. 2, e1501814 (2016).

${ }^{49}$ D. Phelan, C. Stock, J. A. Rodriguez-Rivera, S. X. Chi, J. Leão, X. F. Long, Y. J. Xie, A. A. Bokov, Z. G. Ye, P. Ganesh, and P. M. Gehring, Proc. Natl. Acad. Sci. U. S. A. 111, 1754 (2014).

${ }^{50}$ F. Li, S. J. Zhang, D. Damjanovic, L. Q. Chen, and T. R. Shrout, Adv. Funct. Mater. 28, 1801504 (2018).

${ }^{51}$ F. Li, S. J. Zhang, T. N. Yang, Z. Xu, N. Zhang, G. Liu, J. J. Wang, J. L. Wang, Z. X. Cheng, Z. G. Ye, J. Luo, T. R. Shrout, and L. Q. Chen, Nat. Commun. 7, 13807 (2016).

${ }^{52}$ A. A. Bokov and Z. G. Ye, J. Mater. Sci 41, 31 (2006).

${ }^{53}$ A. A. Bokov and Z. G. Ye, J. Adv. Diel 02, 1241010 (2012).

${ }^{54}$ G. A. Smolenskii, J. Phys. Soc. Jpn 28, 26 (1970).
${ }^{55}$ V. V. Kirillov and V. A. Isupov, Ferroelectrics 5, 3 (1973).

${ }^{56}$ T. Hino, T. Sugioka, S. Tamura, M. Nishida, and T. Araki, J. Laser Micro Nanoeng. 6, 119 (2011).

${ }^{57}$ K. Uchino and S. Nomura, Ferroelectrics 44, 55 (1982).

${ }^{58}$ J. Tholence, J. Appl. Phys. 50, 7369 (1979).

${ }^{59}$ D. Viehland, S. J. Jang, L. E. Cross, and M. Wuttig, J. Appl. Phys. 68, 2916 (1990).

${ }^{60}$ A. K. Tagantsev, Phys. Rev. Lett. 72, 1100 (1994).

${ }^{61}$ A. A. Bokov and Z. G. Ye, J. Phys.: Condens. Matter 12, L541 (2000).

${ }^{62}$ A. E. Glazounov and A. K. Tagantsev, Appl. Phys. Lett. 73, 856 (1998).

${ }^{63}$ Q. Li, S. Danilkin, G. Deng, Z. Li, R. L. Withers, Z. Xu, and Y. Liu, J. Materiomics 4, 345 (2018).

${ }^{64}$ C. W. Tai and K. Z. Baba-Kishi, Acta Mater 54, 5631 (2006).

${ }^{65}$ G. Xu, Z. Zhong, Y. Bing, Z. G. Ye, and G. Shirane, Nat. Mater 5, 134 (2006).

${ }^{66}$ G. Xu, J. Wen, C. Stock, and P. M. Gehring, Nat. Mater 7, 562 (2008).

${ }^{67}$ G. Xu, H. Luo, H. Xu, and Z. Yin, Phys. Rev. B 64, 020102 (2001).

${ }^{68}$ N. Zhang, H. Yokota, A. M. Glazer, Z. Ren, D. Keen, D. S. Keeble, P. A. Thomas, and Z.-G. Ye, Nat. Commun. 5, 5231 (2014).

${ }^{69}$ F. Li, S. J. Zhang, Z. Xu, X. Wei, J. Luo, and T. R. Shrout, J. Appl. Phys. 108, 034106 (2010).

${ }^{70}$ W. Chaisan, R. Yimnirun, S. Ananta, and D. P. Cann, Mater. Lett 59, 3732 (2005).

${ }^{71}$ C. R. Foschini, J. F. Fernández, B. D. Stojanovic, and J. A. Varela, The Effect of Microstructure on the Electrical Properties of PZT Thin Films, in Morphotropic Phase Boundary Perovskites, High Strain Piezoelectrics, and Dielectric Ceramics (Wiley, 2014).

${ }^{72}$ C. R. Foschini, J. F. Li, C. T. A. Suchicital, D. Viehland, B. D. Stojanovic, and J. A. Varela, "Temperature and stoichiometry effect on microstructural and ferroelectric properties of $\mathrm{Pb}\left(\mathrm{Zr}_{1-\mathrm{x}} \mathrm{Ti}_{\mathrm{x}}\right) \mathrm{O}_{3}$ thin films prepared by chemical solution deposition," in Ceramic Materials and Multilayer Electronic Devices (Wiley, 2012).

${ }^{73}$ T. Bongkarn, N. Vittayakorn, and G. Rujijanagul, NU Int. J. Sci. 2, 21 (2005).

${ }^{74}$ C. J. Stringer, R. E. Eitel, T. R. Shrout, C. A. Randall, and I. M. Reaney, J. Appl. Phys 97, 024101 (2005).

${ }^{75}$ J. Guyonnet, Ferroelectric Domain Walls: Statics, Dynamics, and Functionalities Revealed by Atomic Force Microscopy (Springer, Switzerland, 2014).

${ }^{76}$ S. Zhang, J. Luo, W. Hackenberger, and T. R. Shrout, J. Appl. Phys 104, 064106 (2008).

77 IEEE standard on piezoelectricity. ANSI/IEEE. "Report No. 176-1987" (IEEE, New York, 1987).

${ }^{78}$ P. L. Lee, D. Shu, M. Ramanathan, C. Preissner, J. Wang, M. A. Beno, R. B. Von Dreele, L. Ribaud, C. Kurtz, S. M. Antao, X. Jiao, and B. H. Toby, J. Synchrotron Radiat 15, 427 (2008).

79 General Structure Analysis System (GSAS). Los Alamos National Laboratory. “Report LAUR 86-748," (2004).

${ }^{80} \mathrm{G}$. Kresse and J. Furthmüller, Phys. Rev. B 54, 11169 (1996).

${ }^{81}$ G. Liu, J. Gong, L. P. Kong, R. D. Schaller, Q. Hu, Z. Liu, S. Yan, W. G. Yang, C. C. Stoumpos, M. G. Kanatzidis, H.-k. Mao, and T. Xu, Proc. Natl. Acad. Sci. U. S. A. 115, 8076 (2018).

${ }^{82}$ P. E. Blöchl, Phys. Rev. B 50, 17953 (1994).

${ }^{83}$ J. P. Perdew, K. Burke, and M. Ernzerhof, Phys. Rev. Lett. 77, 3865 (1996).

${ }^{84}$ G. I. Csonka, J. P. Perdew, A. Ruzsinszky, P. H. T. Philipsen, S. Lebègue, J. Paier, O. A. Vydrov, and J. G. Ángyán, Phys. Rev. B 79, 155107 (2009).

${ }^{85}$ S. J. Grimme, Comput. Chem. 27, 1787 (2006). 